\title{
Development of Novobiocin Analogues that Manifest Anti-proliferative Activity Against Several Cancer Cell Lines
}

\author{
Joseph a. Burlison, Chris Avila, George Vielhauer, Jeffrey Holzbeierlein, and Brian \\ S.J. Blagg
}

Experimental Procedures 
Experimental for Compound 7

Experimental for Compound 8

Experimental for Compound 12 
Experimental for Compound 33

S22

Experimental for Compound 34

$\mathrm{S} 23$

Experimental for Compound 35

S24

Experimental for Compound 36

S24

Experimental for Compound 37

S25

Experimental for Compound 38

S25

Experimental for Compound 40

S26

Experimental for Compound 41

S26

Experimental for Compound 42

S27

Experimental for Compound 43

S27

Experimental for Compound 44

S28

Experimental for Compound 45

S28

Experimental for Compound 46

S29

Experimental for Compound 47

S29 
3-Amino-7-((3aR, 4R, 7R, 7aR)-7-methoxy-6,6-dimethyl-2-oxo oxotetrahydrdo-3aH[1.3]dioxolo[4,5-c]pyran-4-yloxy)-8-methyl-2H-chromen-2-one (7). Palladium on carbon (10\%, 67 mg) was added to a solution of carbamate $5(670 \mathrm{mg}, 1.31 \mathrm{mmol})$ in THF $(13 \mathrm{~mL})$. The suspension was stirred for $6 \mathrm{~h}$ under a hydrogen atmosphere before it was filtered through a plug of $\mathrm{SiO}_{2}$ and eluted with THF (20 $\mathrm{mL})$. The eluent was concentrated and the residue purified by chromatography $\left(\mathrm{SiO}_{2}, 100: 1 \rightarrow 50: 1\right.$; $\mathrm{CH}_{2} \mathrm{Cl}_{2}$ :acetone) to afford $6(425 \mathrm{mg}, 83 \%)$ as a yellow foam: $[\alpha]^{23}{ }_{\mathrm{D}}=-26.4^{\circ}(\mathrm{c}=0.780,20 \% \mathrm{MeOH}$ in $\left.\mathrm{CH}_{2} \mathrm{Cl}_{2}\right) ;{ }^{1} \mathrm{H} \mathrm{NMR}\left(\mathrm{CDCl}_{3}, 400 \mathrm{MHz}\right) \delta 7.10(\mathrm{~d}, J=8.6 \mathrm{~Hz}, 1 \mathrm{H}), 7.05(\mathrm{~d}, J=8.6 \mathrm{~Hz}, 1 \mathrm{H}), 6.68(\mathrm{~s}, 1 \mathrm{H})$, $5.73(\mathrm{~d}, J=2.0 \mathrm{~Hz}, 1 \mathrm{H}), 5.04(\mathrm{dd}, J=2.0,7.9 \mathrm{~Hz}, 1 \mathrm{H}), 4.95(\mathrm{t}, J=7.7 \mathrm{~Hz}, 1 \mathrm{H}), 4.11(\mathrm{~s}, 2 \mathrm{H}), 3.54(\mathrm{~s}, 3 \mathrm{H})$, $3.29(\mathrm{~d}, J=7.6 \mathrm{~Hz}, 1 \mathrm{H}), 2.28(\mathrm{~s}, 3 \mathrm{H}), 1.34(\mathrm{~s}, 3 \mathrm{H}), 1.21(\mathrm{~s}, 3 \mathrm{H}) ;{ }^{13} \mathrm{C} \mathrm{NMR}\left(\mathrm{CDCl}_{3}, 200 \mathrm{MHz}\right) \delta 159.6$, $153.3,153.0,148.1,130.2,122.7,116.1,114.8,111.9,111.0,94.5,83.0,78.0,77.3,76.4,60.6,27.5,22.2$, 8.6; IR (film) $v_{\max } 3462,3362,2984,2937,2839,1807,1707,1636,1595,1497,1387,1371,1331,1263$, 1169, 1109, 1078, $1036 \mathrm{~cm}^{-1}$; HRMS (ESI+) m/z $392.1357\left(\mathrm{M}+\mathrm{H}^{+}, \mathrm{C}_{19} \mathrm{H}_{22} \mathrm{NO}_{8}\right.$ requires $m / z$ 392.1346).

General EDCI coupling procedure A: $N$-(3-Dimethylamino-propyl)- $N$ '-ethylcarbodiimide hydrochloride (3 eq) was added to a solution of aminocoumarin 7 (1 eq), benzoic acid (3 eq) and 4-DMAP (2.0 eq) in $\mathrm{CH}_{2} \mathrm{Cl}_{2}$ at $\mathrm{rt}$. The solution was stirred for $14 \mathrm{~h}$, concentrated and the residue purified via preparative TLC or column chrotography $\left(\mathrm{SiO}_{2}, 40: 1 ; \mathrm{CH}_{2} \mathrm{Cl}_{2}\right.$ :acetone) to afford the benzamide.

7a

Benzyl-7-((3aR, 4R, 7R, 7aR)-7-methoxy-6,6-dimethyl-2-oxotetrahydrdo-3aH[1.3]dioxolo[4,5-c]pyran-4-yloxy)-8-methyl-2-oxo-2H-chromen-3-ylcarbamate (7a). Boron trifluoride etherate $(61 \mu \mathrm{L}, 69 \mathrm{mg}, 0.49 \mathrm{mmol}, 30 \mathrm{~mol} \%)$ was added dropwise to a solution of (3aR, 4S, 7R, 7aR)-7methoxy-6,6-dimethyl-2-oxo-tetrahydro-3aH-[1,3]dioxolo[4,5-c]pyran-4-yl 2,2,2-trichloroacetimidate ${ }^{1,2}$ (6, $588 \mathrm{mg}, 1.62 \mathrm{mmol}$ ) and benzyl 7-hydroxy-8-methyl-2-oxo-2H-chromen-3-yl carbamate ${ }^{3}(5,527 \mathrm{mg}, 1.62$ $\mathrm{mmol})$ in $\mathrm{CH}_{2} \mathrm{Cl}_{2}(16 \mathrm{~mL})$ at $\mathrm{rt}$. The mixture was stirred for $14 \mathrm{~h}$, before $\mathrm{Et}_{3} \mathrm{~N}(150 \mu \mathrm{L})$ was added and the mixture concentrated. The residue was purified by chromatography $\left(\mathrm{SiO}_{2}, \mathrm{DCM} \rightarrow 100: 1 ; \mathrm{CH}_{2} \mathrm{Cl}_{2}:\right.$ acetone $)$ to afford 7a $(670 \mathrm{mg}, 81 \%)$ as a yellow foam: $[\alpha]_{\mathrm{D}}^{22}=-19.7^{\circ}\left(\mathrm{c}=1.54,20 \% \mathrm{MeOH}\right.$ in $\left.\mathrm{CH}_{2} \mathrm{Cl}_{2}\right) ;{ }^{1} \mathrm{H} \mathrm{NMR}^{\mathrm{N}}$ $\left(\mathrm{CDCl}_{3}, 400 \mathrm{MHz}\right) \delta 8.27(\mathrm{~s}, 1 \mathrm{H}), 7.85(\mathrm{~s}, 1 \mathrm{H}), 7.55-7.35(\mathrm{~m}, 5 \mathrm{H}), 7.29(\mathrm{~d}, J=2.9 \mathrm{~Hz}, 1 \mathrm{H}), 7.11(\mathrm{~d}, J=$ $8.7 \mathrm{~Hz}, 1 \mathrm{H}), 5.77(\mathrm{~d}, J=1.9 \mathrm{~Hz}, 1 \mathrm{H}), 5.23(\mathrm{~s}, 2 \mathrm{H}), 5.05(\mathrm{~d}, J=1.9 \mathrm{~Hz}, 1 \mathrm{H}), 4.95(\mathrm{t}, J=7.7 \mathrm{~Hz}, 1 \mathrm{H}), 3.59$ $(\mathrm{s}, 3 \mathrm{H}), 3.30(\mathrm{~d}, J=7.6 \mathrm{~Hz}, 1 \mathrm{H}), 2.27(\mathrm{~s}, 3 \mathrm{H}), 1.34(\mathrm{~s}, 3 \mathrm{H}), 1.19(\mathrm{~s}, 3 \mathrm{H}) ;{ }^{13} \mathrm{C} \mathrm{NMR}\left(\mathrm{CDCl}_{3}, 100 \mathrm{MHz}\right) \delta$ 159.0, 155.2, 153.6, 153.6, 149.2, 136.0, 129.1 (2C), 129.0, 128.7 (2C), 125.8, 122.6, 122.1, 115.2, 115.1, 111.6, 94.8, 83.3, 78.4, 77.6, 77.0, 67.9, 61.0, 27.9, 22.6, 8.8; IR (film) $v_{\max } 3402,3319,3063,3034,2984$, 2939, 2839, 1817, 1709, 1634, 1609, 1587, 1522, 1456, 1383, 1366, 1331, 1296, 1263, 1229, 1205,1175

$\mathrm{cm}^{-1}$; HRMS (ESI+) m/z 526.1688 $\left(\mathrm{M}+\mathrm{H}^{+}, \mathrm{C}_{27} \mathrm{H}_{28} \mathrm{NO}_{10}\right.$ requires $\left.\mathrm{m} / \mathrm{z} 526.1713\right)$. 


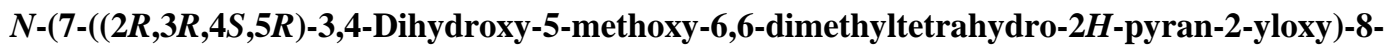
methyl-2-oxo-2H-chromen-3-yl)benzamide (8). Colorless solid $(83 \%)$ : $[\alpha]_{\mathrm{D}}^{24}=-25.2^{\circ}(\mathrm{c}=0.16,20 \%$ $\mathrm{MeOH}$ in $\left.\mathrm{CH}_{2} \mathrm{Cl}_{2}\right) ;{ }^{1} \mathrm{H}$ NMR (400 MHz, 20\% $\mathrm{CD}_{3} \mathrm{OD}$ in $\left.\mathrm{CD}_{2} \mathrm{Cl}_{2}\right) \delta 8.77(\mathrm{~s}, 1 \mathrm{H}), 7.89(\mathrm{~d}, J=7.4 \mathrm{~Hz}, 2 \mathrm{H})$ 7.59 (t, $J=7.4 \mathrm{~Hz}, 1 \mathrm{H}), 7.53$ (d, $J=6.3 \mathrm{~Hz}, 1 \mathrm{H}), 7.51$ (d, $J=7.4 \mathrm{~Hz}, 1 \mathrm{H}), 7.39$ (d, J=8.7 Hz, $1 \mathrm{H}), 7.21$ (d, $J=8.7 \mathrm{~Hz}, 1 \mathrm{H}), 5.55(\mathrm{~d}, J=2.2 \mathrm{~Hz}, 1 \mathrm{H}), 4.18-4.08(\mathrm{~m}, 2 \mathrm{H}), 3.57(\mathrm{~s}, 3 \mathrm{H}), 3.33-3.31(\mathrm{~m}, 1 \mathrm{H}), 2.27(\mathrm{~s}$, $3 \mathrm{H}), 1.35(\mathrm{~s}, 3 \mathrm{H}), 1.10(\mathrm{~s}, 3 \mathrm{H}) ;{ }^{13} \mathrm{C} \mathrm{NMR}\left(100 \mathrm{MHz}, 20 \% \mathrm{CD}_{3} \mathrm{OD}\right.$ in $\left.\mathrm{CD}_{2} \mathrm{Cl}_{2}\right) \delta 164.5,157.7,154.6,147.6$, 132.1, 130.7, 127.2 (2C), 125.5 (2C), 124.2, 123.1, 120.0, 112.5, 112.1, 110.0, 96.7, 82.5, 76.9, 69.5, 66.8, 60.0, 27.0, 20.6, 6.3; IR (film) $v_{\max } 3400,3088,3065,2978,2926,2853,1713,1668,1607,1526,1493$, 1369, 1252, 1092, $1080 \mathrm{~cm}^{-1}$; HRMS (ESI+) m/z 470.1826 (M+ $\mathrm{H}^{+}, \mathrm{C}_{25} \mathrm{H}_{28} \mathrm{NO}_{8}$ requires $m / z$ 470.1815).<smiles>CO[C@H]1[C@@H](O)[C@@H](O)[C@H](Oc2ccc3cc(NC(=O)c4ccccc4)c(=O)oc3c2C)OC1(C)C</smiles><smiles>COc1ccccc1C(=O)Nc1cc2ccc(O[C@@H]3OC(C)(C)[C@H](OC)[C@H](O)[C@H]3O)c(C)c2oc1=O</smiles>

9

$N$-(7-((2R,3R,4S,5R)-3,4-Dihydroxy-5-methoxy-6,6-dimethyltetrahydro-2H-pyran-2-yloxy)-8methyl-2-oxo-2H-chromen-3-yl)-2-methoxybenzamide (9). Colorless solid $(74 \%):[\alpha]_{\mathrm{D}}^{25}=-16.1^{\mathrm{o}}(\mathrm{c}=$ 0.16, 20\% $\mathrm{MeOH}$ in $\left.\mathrm{CH}_{2} \mathrm{Cl}_{2}\right) ;{ }^{1} \mathrm{H} \mathrm{NMR}\left(400 \mathrm{MHz}, 20 \% \mathrm{CD}_{3} \mathrm{OD}\right.$ in $\left.\mathrm{CD}_{2} \mathrm{Cl}_{2}\right) \delta 8.79(\mathrm{~s}, 1 \mathrm{H}), 8.15(\mathrm{~d}, J=7.9$ $\mathrm{Hz}, 1 \mathrm{H}), 7.57$ (t, $J=7.8 \mathrm{~Hz}, 1 \mathrm{H}), 7.38(\mathrm{~d}, J=8.7 \mathrm{~Hz}, 1 \mathrm{H}), 7.20$ (d, J=8.7 Hz, 1H), $7.16-7.10$ (m, 2H), $5.55(\mathrm{~s}, 1 \mathrm{H}) 4.20-4.14(\mathrm{~m}, 2 \mathrm{H}), 4.11(\mathrm{~s}, 3 \mathrm{H}), 3.58(\mathrm{~s}, 3 \mathrm{H}), 3.35(\mathrm{~d}, J=8.0 \mathrm{~Hz}, 1 \mathrm{H}), 2.28(\mathrm{~s}, 3 \mathrm{H}), 1.34(\mathrm{~s}$, $3 \mathrm{H}), 1.11(\mathrm{~s}, 3 \mathrm{H}) ;{ }^{13} \mathrm{C} \mathrm{NMR}\left(100 \mathrm{MHz}, 20 \% \mathrm{CD}_{3} \mathrm{OD}\right.$ in $\left.\mathrm{CD}_{2} \mathrm{Cl}_{2}\right) \delta 164.9,160.2,158.4,156.7,149.8$, 134.6, 132.4, 126.3, 125.3, 123.0, 122.9, 121.9, 121.2, 114.7, 112.5, 111.7, 99.1, 84.8, 79.2, 71.9, 69.0, 62.1, 56.8, 29.1, 22.9, 8.5; IR (film) $v_{\max } 3373,2947,2835,2525,1641,1630,1610,1448,1412,1398 \mathrm{~cm}^{-}$ 1. HRMS (ESI+) $m / z 500.1893\left(\mathrm{M}+\mathrm{H}^{+}, \mathrm{C}_{26} \mathrm{H}_{30} \mathrm{NO}_{9}\right.$ requires $m / z$ 500.1921).<smiles>COc1ccccc1C(=O)Nc1cc2ccc(O[C@@H]3OC(C)(C)[C@H](OC)[C@H]4OC(=O)O[C@@H]34)c(C)c2oc1=O</smiles> 
9a

2-Methoxy-N-(7-((3aR,4R,7R,7aR)-7-methoxy-6,6-dimethyl-2-oxotetrahydro-3aH-

[1,3]dioxolo[4,5-c]pyran-4-yloxy)-8-methyl-2-oxo-2H-chromen-3-yl)benzamide (9a). Colorless solid $(66 \%):[\alpha]^{24}=-29.6^{\circ}\left(\mathrm{c}=0.61,20 \% \mathrm{MeOH}\right.$ in $\left.\mathrm{CH}_{2} \mathrm{Cl}_{2}\right) ;{ }^{1} \mathrm{H} \mathrm{NMR}\left(400 \mathrm{MHz}, \mathrm{CDCl}_{3}\right) \delta 8.77(\mathrm{~s}, 1 \mathrm{H})$, $8.18(\mathrm{~d}, J=7.8 \mathrm{~Hz}, 1 \mathrm{H}), 7.46(\mathrm{t}, J=7.8 \mathrm{~Hz}, 1 \mathrm{H}), 7.27(\mathrm{~d}, J=8.3 \mathrm{~Hz}, 1 \mathrm{H}), 7.09-7.02(\mathrm{~m}, 2 \mathrm{H}), 6.99(\mathrm{~d}, J=$ $8.3 \mathrm{~Hz}, 1 \mathrm{H}), 5.71(\mathrm{~s}, 1 \mathrm{H}), 4.99(\mathrm{~d}, J=7.9 \mathrm{~Hz}, 1 \mathrm{H}), 4.91(\mathrm{t}, J=7.9 \mathrm{~Hz}, 1 \mathrm{H}), 4.05(\mathrm{~s}, 3 \mathrm{H}), 3.53(\mathrm{~s}, 3 \mathrm{H}), 3.24$ $(\mathrm{d}, J=7.9 \mathrm{~Hz}, 1 \mathrm{H}), 2.23(\mathrm{~s}, 3 \mathrm{H}), 1.29(\mathrm{~s}, 3 \mathrm{H}), 1.14(\mathrm{~s}, 3 \mathrm{H}) ;{ }^{13} \mathrm{C} \mathrm{NMR}\left(100 \mathrm{MHz}, \mathrm{CDCl}_{3}\right) \delta 164.5,159.6$, $158.1,155.3,153.6,149.5,134.3,132.6,126.4,124.4,123.5,121.9,121.3,115.5,115.1,112.0,111.5$, 94.8, 83.3, 78.4, 77.4, 77.1, 61.0, 56.6, 27.9, 22.6, 8.8; IR (film) $v_{\max } 3308,3055,2986,2939,2930,1817$, 1807, 1707, 1655, 1603, 1533, 1481, 1466, 1367, 1263, $1232 \mathrm{~cm}^{-1}$; HRMS (ESI+) m/z 526.1691 (M + H , $\mathrm{C}_{27} \mathrm{H}_{28} \mathrm{NO}_{10}$ requires $\mathrm{m} / \mathrm{z} 526.1713$ ).<smiles>COc1cccc(C(=O)Nc2cc3ccc(O[C@@H]4OC(C)(C)[C@@H](OC)[C@@H](O)[C@H]4O)c(C)c3oc2=O)c1</smiles>

10

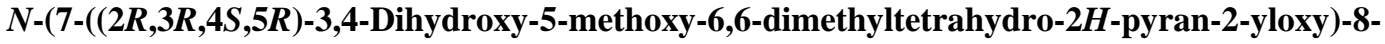
methyl-2-oxo-2H-chromen-3-yl)-3-methoxybenzamide (10). Colorless solid $(59 \%):[\alpha]^{24}=-16.9^{\circ}(\mathrm{c}=$ 0.81, 20\% $\mathrm{MeOH}$ in $\mathrm{CH}_{2} \mathrm{Cl}_{2}$ ); ${ }^{1} \mathrm{H}$ NMR (400 MHz, 20\% $\mathrm{CD}_{3} \mathrm{OD}$ in $\left.\mathrm{CD}_{2} \mathrm{Cl}_{2}\right) \delta 8.70(\mathrm{~s}, 1 \mathrm{H}), 7.42-7.33(\mathrm{~m}$, 4H), $7.19(\mathrm{~d}, J=8.7 \mathrm{~Hz}, 1 \mathrm{H}), 7.13(\mathrm{~d}, J=8.7 \mathrm{~Hz}, 1 \mathrm{H}), 5.52(\mathrm{~s}, 1 \mathrm{H}), 4.19-4.05(\mathrm{~m}, 2 \mathrm{H}), 3.84$ (s, $3 \mathrm{H}), 3.55$ $(\mathrm{s}, 3 \mathrm{H}), 3.32(\mathrm{~d}, J=7.8 \mathrm{~Hz}, 1 \mathrm{H}), 2.24(\mathrm{~s}, 3 \mathrm{H}), 1.30(\mathrm{~s}, 3 \mathrm{H}), 1.07(\mathrm{~s}, 3 \mathrm{H}) ;{ }^{13} \mathrm{C} \mathrm{NMR}(100 \mathrm{MHz}, 20 \%$ $\mathrm{CD}_{3} \mathrm{OD}$ in $\left.\mathrm{CD}_{2} \mathrm{Cl}_{2}\right) \delta 167.1,160.8,160.1,157.1,150.0,135.9,130.6,126.5,126.1,122.2,119.6,118.9$, 114.8, 114.4, 113.3, 111.9, 99.2, 84.8, 79.3, 71.9, 69.1, 62.1, 56.0, 29.1, 22.9, 8.4; IR (film) $v_{\max } 3400$, 3082, 2980, 2937, 2835, 1709, 1670, 1607, 1526, 1369, 1259, 1138, $1090 \mathrm{~cm}^{-1}$; HRMS (ESI+) m/z $500.1899\left(\mathrm{M}+\mathrm{H}^{+}, \mathrm{C}_{26} \mathrm{H}_{30} \mathrm{NO}_{9}\right.$ requires $m / z$ 500.1921).<smiles>COc1cccc(C(=O)Nc2cc3ccc(O[C@@H]4OC(C)(C)C(OC)[C@H]5OC(=O)O[C@H]45)c(C)c3oc2=O)c1</smiles>

$10 a$

3-Methoxy-N-(7-((3aR,4R,7R,7aR)-7-methoxy-6,6-dimethyl-2-oxotetrahydro-3aH[1,3]dioxolo[4,5-c]pyran-4-yloxy)-8-methyl-2-oxo-2H-chromen-3-yl)benzamide (10a). Colorless solid 
(43\%): $[\alpha]^{25}=-27.1^{\circ}\left(\mathrm{c}=1.22,20 \% \mathrm{MeOH}\right.$ in $\left.\mathrm{CH}_{2} \mathrm{Cl}_{2}\right) ;{ }^{1} \mathrm{H}$ NMR $\left(400 \mathrm{MHz}, \mathrm{CDCl}_{3}\right) \delta 8.82(\mathrm{~s}, 1 \mathrm{H})$, $8.76(\mathrm{~s}, 1 \mathrm{H}), 7.51-7.44(\mathrm{~m}, 3 \mathrm{H}), 7.39(\mathrm{~d}, J=8.7 \mathrm{~Hz}, 1 \mathrm{H}), 7.19-7.11(\mathrm{~m}, 2 \mathrm{H}), 5.81(\mathrm{~d}, J=1.8 \mathrm{~Hz}, 1 \mathrm{H})$, 5.08 (dd, $J=1.8,7.8 \mathrm{~Hz}, 1 \mathrm{H}), 4.98(\mathrm{t}, J=7.8 \mathrm{~Hz}, 1 \mathrm{H}), 3.91$ (s, 3H), $3.62(\mathrm{~s}, 3 \mathrm{H}), 3.33$ (d, $J=7.8 \mathrm{~Hz}, 1 \mathrm{H})$, $2.32(\mathrm{~s}, 3 \mathrm{H}), 1.38(\mathrm{~s}, 3 \mathrm{H}), 1.22(\mathrm{~s}, 3 \mathrm{H}) ;{ }^{13} \mathrm{C}$ NMR $\left(100 \mathrm{MHz}, \mathrm{CDCl}_{3}\right) \delta 166.3,160.5,159.6,155.6,153.6$, $149.5,135.5,130.4,126.3,124.5,122.6,119.3,119.0,115.3,115.1,112.9,111.7,94.8,83.3,78.3,77.4$, 77.1, 61.0, 55.9, 27.9, 22.6, 8.9; IR (film) $v_{\max } 3402,3057,2988,2939,2839,1819,1809,1709,1676$, 1609, 1526, 1487, 1369, 1263, 1175, 1153, 1097, $1076 \mathrm{~cm}^{-1}$; HRMS (ESI +$) \mathrm{m} / \mathrm{z} 526.1695\left(\mathrm{M}+\mathrm{H}^{+}\right.$, $\mathrm{C}_{27} \mathrm{H}_{28} \mathrm{NO}_{10}$ requires $\mathrm{m} / \mathrm{z}$ 526.1713).

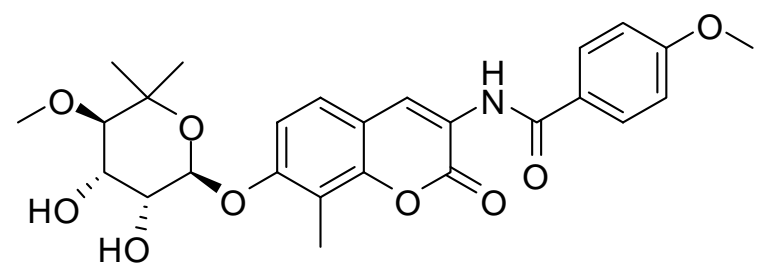

11

$N$-(7-((2R,3R,4S,5R)-3,4-Dihydroxy-5-methoxy-6,6-dimethyltetrahydro-2H-pyran-2-yloxy)-8methyl-2-oxo-2H-chromen-3-yl)-4-methoxybenzamide (11). Colorless solid (75\%): $[\alpha]_{\mathrm{D}}^{25}=-13.0^{\circ}(\mathrm{c}=$ 0.10, 20\% MeOH in $\mathrm{CH}_{2} \mathrm{Cl}_{2}$ ); ${ }^{1} \mathrm{H}$ NMR (400 MHz, $\mathrm{CD}_{2} \mathrm{Cl}_{2}$ ) $\delta 8.78$ (s, $\left.1 \mathrm{H}\right), 8.68$ (s, $\left.1 \mathrm{H}\right), 7.91$ (dt, $J=2.6$, $6.9 \mathrm{~Hz}, 2 \mathrm{H}), 7.40$ (d, $J=8.7 \mathrm{~Hz}, 1 \mathrm{H}), 7.22(\mathrm{~d}, J=8.7 \mathrm{~Hz}, 1 \mathrm{H}), 7.03$ (dt, $J=2.6,8.9 \mathrm{~Hz}, 2 \mathrm{H}), 5.63$ (d, $J=$ $2.0 \mathrm{~Hz}, 1 \mathrm{H}), 4.30-4.23(\mathrm{~m}, 2 \mathrm{H}), 3.91(\mathrm{~s}, 3 \mathrm{H}), 3.62(\mathrm{~s}, 3 \mathrm{H}), 3.38(\mathrm{~d}, J=8.8 \mathrm{~Hz}, 1 \mathrm{H}), 2.98-2.70(\mathrm{~m}, 2 \mathrm{H})$, $2.31(\mathrm{~s}, 3 \mathrm{H}), 1.39$ (s, 3H), $1.16(\mathrm{~s}, 3 \mathrm{H}) ;{ }^{13} \mathrm{C}$ NMR (100 MHz, $\left.\mathrm{CD}_{2} \mathrm{Cl}_{2}\right) \delta 165.7,163.3,159.7,156.3,149.4$ (2C), 129.4, 126.3, 126.0, 124.2, 122.4, 114.5 (2C), 114.4 (2C), 111.5, 98.3, 84.6, 78.9, 71.5, 68.9, 62.1, 55.9, 29.2, 22.7, 8.5; IR (film) $v_{\max } 3404,2976,2934,2841,1607,1506,1369,1248,1176,1091 \mathrm{~cm}^{-1}$; HRMS (ESI+) m/z $500.1896\left(\mathrm{M}+\mathrm{H}^{+}, \mathrm{C}_{26} \mathrm{H}_{30} \mathrm{NO}_{9}\right.$ requires $m / z$ 500.1921).

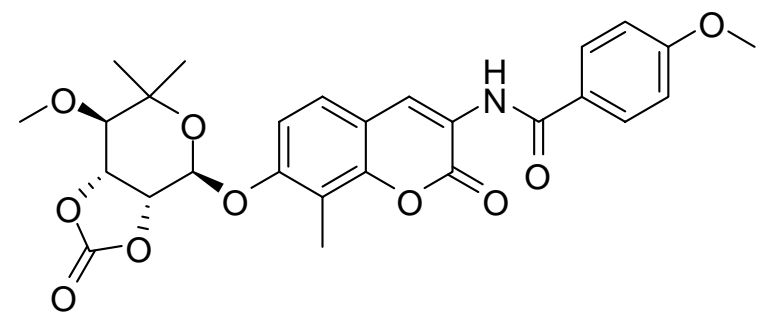

11a

4-Methoxy-N-(7-((3aR,4R,7R,7aR)-7-methoxy-6,6-dimethyl-2-oxotetrahydro-3aH-

[1,3]dioxolo[4,5-c]pyran-4-yloxy)-8-methyl-2-oxo-2H-chromen-3-yl)benzamide (11a). Colorless solid $(35 \%):[\alpha]^{24}=-25.8^{\circ}\left(\mathrm{c}=0.69,20 \% \mathrm{MeOH}\right.$ in $\left.\mathrm{CH}_{2} \mathrm{Cl}_{2}\right) ;{ }^{1} \mathrm{H} \mathrm{NMR}\left(400 \mathrm{MHz}, \mathrm{CDCl}_{3}\right) \delta 8.81(\mathrm{~s}, 1 \mathrm{H})$, $8.71(\mathrm{~s}, 1 \mathrm{H}), 7.91(\mathrm{~d}, J=8.8 \mathrm{~Hz}, 2 \mathrm{H}), 7.38(\mathrm{~d}, J=8.7 \mathrm{~Hz}, 1 \mathrm{H}), 7.16(\mathrm{~d}, J=8.7 \mathrm{~Hz}, 1 \mathrm{H}), 7.02(\mathrm{~d}, J=8.8$ $\mathrm{Hz}, 2 \mathrm{H}), 5.81$ (d, $J=1.8 \mathrm{~Hz}, 1 \mathrm{H}), 5.08(\mathrm{dd}, J=1.9,7.9 \mathrm{~Hz}, 1 \mathrm{H}), 4.98$ (t, $J=7.8 \mathrm{~Hz}, 1 \mathrm{H}), 3.91(\mathrm{~s}, 3 \mathrm{H}), 3.62$ 
(s, 3H), $3.33(\mathrm{~d}, J=7.8 \mathrm{~Hz}, 1 \mathrm{H}), 2.35(\mathrm{~s}, 3 \mathrm{H}), 1.38(\mathrm{~s}, 3 \mathrm{H}), 1.23(\mathrm{~s}, 3 \mathrm{H}) ;{ }^{13} \mathrm{C}$ NMR $\left(100 \mathrm{MHz}, \mathrm{CDCl}_{3}\right) \delta$ 165.9, 163.4, 159.7, 155.5, 153.6, 149.4, 129.5 (3C), 126.3, 126.2, 124.1, 122.8, 115.3, 114.5 (2C), 111.7, 94.8, 83.3, 78.3, 77.4, 77.1, 61.0, 55.9, 27.9, 22.6, 8.9; IR (film) $v_{\max } 3406,2984,2937,2839,1811,1709$, 1670, 1607, 1529, 1506, 1367, 1246, $1175 \mathrm{~cm}^{-1}$; HRMS (ESI+) $\mathrm{m} / \mathrm{z} 526.1690\left(\mathrm{M}+\mathrm{H}^{+}, \mathrm{C}_{27} \mathrm{H}_{28} \mathrm{NO}_{10}\right.$ requires $m / z 526.1713)$.<smiles>CO[C@H]1[C@@H](O)[C@@H](O)C(Oc2ccc3cc(NC(=O)c4ccccc4-c4ccccc4)c(=O)oc3c2C)OC1(C)C</smiles>

12

$N$-(7-((2R,3R,4S,5R)-3,4-Dihydroxy-5-methoxy-6,6-dimethyltetrahydro-2H-pyran-2-yloxy)-8methyl-2-oxo-2H-chromen-3-yl)biphenyl-2-carboxamide (12). Colorless solid (55\%): $[\alpha]_{\mathrm{D}}^{24}=-7.2^{\circ}(\mathrm{c}$ $=0.13,20 \% \mathrm{MeOH}$ in $\left.\mathrm{CH}_{2} \mathrm{Cl}_{2}\right) ;{ }^{1} \mathrm{H} \operatorname{NMR}\left(400 \mathrm{MHz}, \mathrm{CD}_{2} \mathrm{Cl}_{2}\right) \delta 8.77(\mathrm{~s}, 1 \mathrm{H}), 7.99(\mathrm{~s}, 1 \mathrm{H}), 7.78(\mathrm{~d}, J=8.0$ $\mathrm{Hz}, 1 \mathrm{H}), 7.65-7.33(\mathrm{~m}, 9 \mathrm{H}), 7.20$ (d, J = 8.8 Hz, 1H), 5.61 (d, J=1.8 Hz, 1H), $4.28-4.21(\mathrm{~m}, 2 \mathrm{H}), 3.61$ (s, 3H), $3.37(\mathrm{~d}, J=8.7 \mathrm{~Hz}, 1 \mathrm{H}), 2.25(\mathrm{~s}, 3 \mathrm{H}), 1.38(\mathrm{~s}, 3 \mathrm{H}), 1.14(\mathrm{~s}, 3 \mathrm{H}) ;{ }^{13} \mathrm{C}$ NMR $\left(125 \mathrm{MHz}, \mathrm{CD}_{2} \mathrm{Cl}_{2}\right) \delta$ 168.5, 158.8, 156.3, 149.5, 140.6, 140.2, 135.4, 131.3, 131.1, 129.1 (4C), 129.0, 128.3, 128.0, 126.0, 124.0, $122.1,114.5,114.2,111.4,98.2,84.6,78.8,71.6,68.9,62.1,29.3,22.6,8.4$; IR (film) $v_{\max } 3379,3059$, 2982, 2932, 2831, 1713, 1668, 1607, 1520, 1367, 1258, 1113, $1092 \mathrm{~cm}^{-1}$; HRMS (ESI+) m/z 546.2100 (M $+\mathrm{H}^{+}, \mathrm{C}_{31} \mathrm{H}_{32} \mathrm{NO}_{8}$ requires $\mathrm{m} / \mathrm{z}$ 546.2128).<smiles>CO[C@H]1[C@H]2OC(=O)O[C@H]2[C@H](Oc2ccc3cc(NC(=O)c4ccccc4-c4ccccc4)c(=O)oc3c2C)OC1(C)C</smiles>

12a

$N$-(7-((3aR,4R,7R,7aR)-7-Methoxy-6,6-dimethyl-2-oxotetrahydro-3aH-[1,3]dioxolo[4,5-

clpyran-4-yloxy)-8-methyl-2-oxo-2H-chromen-3-yl)biphenyl-2-carboxamide (12a). Colorless solid $(35 \%):[\alpha]^{26}{ }_{\mathrm{D}}=-22.8^{\circ}\left(\mathrm{c}=0.15,20 \% \mathrm{MeOH}\right.$ in $\left.\mathrm{CH}_{2} \mathrm{Cl}_{2}\right) ;{ }^{1} \mathrm{H} \mathrm{NMR}\left(400 \mathrm{MHz}, \mathrm{CDCl}_{3}\right) \delta 8.70(\mathrm{~s}, 1 \mathrm{H})$, 7.93 (s, 1H), 7.80 (d, $J=7.8 \mathrm{~Hz}, 1 \mathrm{H}), 7.61-7.36$ (m, 8H), 7.33 (d, $J=8.7 \mathrm{~Hz}, 1 \mathrm{H}), 7.13$ (d, $J=8.7 \mathrm{~Hz}$, 1H), 5.79 (d, $J=1.8 \mathrm{~Hz}, 1 \mathrm{H}), 5.6$ (dd, $J=1.8,7.9 \mathrm{~Hz}, 1 \mathrm{H}), 4.97$ (t, $J=7.9 \mathrm{~Hz}, 1 \mathrm{H}), 3.61$ (s, 3H), 3.32 (d, $J$ $=7.9 \mathrm{~Hz}, 1 \mathrm{H}), 2.28(\mathrm{~s}, 3 \mathrm{H}), 1.37(\mathrm{~s}, 3 \mathrm{H}), 1.21(\mathrm{~s}, 3 \mathrm{H}) ;{ }^{13} \mathrm{C} \mathrm{NMR}\left(100 \mathrm{MHz}, \mathrm{CDCl}_{3}\right) \delta 168.8,158.8,155.4$ (2C), 140.6, 140.0, 135.1, 131.5, 131.1, 129.4 (2C), 129.2 (2C), 129.1 (2C), 128.6, 128.2, 126.2, 124.0, 
122.4 115.2, 115.1, 111.5, 94.7, 83.3, 78.3, 77.4, 77.1, 61.0, 27.9, 22.6, 8.8; IR (film) $v_{\max } 3375,2984$, $2935,1815,1715,1672,1609,1516,1367,1256,1171,1111,1094,1076 \mathrm{~cm}^{-1}$; HRMS (ESI +$) \mathrm{m} / \mathrm{z}$ $572.1893\left(\mathrm{M}+\mathrm{H}^{+}, \mathrm{C}_{32} \mathrm{H}_{30} \mathrm{NO}_{9}\right.$ requires $m / z$ 572.1921).<smiles>CO[C@H]1[C@@H](O)[C@@H](O)[C@H](Oc2ccc3cc(NC(=O)c4cccc(-c5ccccc5)c4)c(=O)oc3c2C)OC1(C)C</smiles>

13

$N$-(7-((2R,3R,4S,5R)-3,4-Dihydroxy-5-methoxy-6,6-dimethyltetrahydro-2H-pyran-2-yloxy)-8methyl-2-oxo-2H-chromen-3-yl)biphenyl-3-carboxamide (13). Colorless solid (75\%): $[\alpha]_{\mathrm{D}}^{24}=-24.3^{\circ}$ (c $=0.09,20 \% \mathrm{MeOH}$ in $\left.\mathrm{CH}_{2} \mathrm{Cl}_{2}\right) ;{ }^{1} \mathrm{H}$ NMR $\left(400 \mathrm{MHz}, 20 \% \mathrm{CD}_{3} \mathrm{OD}\right.$ in $\left.\mathrm{CD}_{2} \mathrm{Cl}_{2}\right) \delta 8.84(\mathrm{~s}, 1 \mathrm{H}), 8.82(\mathrm{~s}$, 1H), 8.17 (s, 1H), $7.91(\mathrm{~d}, J=7.7 \mathrm{~Hz}, 1 \mathrm{H}), 7.86$ (t, $J=6.7 \mathrm{~Hz}, 1 \mathrm{H}), 7.71$ (dd, $J=1.3,7.7 \mathrm{~Hz}, 2 \mathrm{H}), 7.63$ (t, $J=7.7 \mathrm{~Hz}, 1 \mathrm{H}), 7.53$ (t, $J=7.7 \mathrm{~Hz}, 2 \mathrm{H}), 7.44$ (t, $J=7.5 \mathrm{~Hz}, 2 \mathrm{H}), 7.25$ (d, $J=8.7 \mathrm{~Hz}, 1 \mathrm{H}), 5.64(\mathrm{~s}, 1 \mathrm{H})$, $4.31-4.25(\mathrm{~m}, 2 \mathrm{H}), 3.62(\mathrm{~s}, 3 \mathrm{H}), 3.38(\mathrm{~d}, J=8.7 \mathrm{~Hz}, 1 \mathrm{H}), 2.32(\mathrm{~s}, 3 \mathrm{H}), 1.39(\mathrm{~s}, 3 \mathrm{H}), 1.16(\mathrm{~s}, 3 \mathrm{H}) ;{ }^{13} \mathrm{C}$ NMR (125 MHz, 20\% $\mathrm{CD}_{3} \mathrm{OD}$ in $\left.\mathrm{CD}_{2} \mathrm{Cl}_{2}\right) \delta 166.9,159.8,156.8,149.7,145.0,142.4,140.3,134.8,131.3$, 129.7, 129.3 (2C), 128.2, 127.6 (2C), 126.2 (2C), 125.7, 121.9, 114.5, 114.1, 111.6, 98.9, 84.5, 78.9, 71.6, 68.7, 61.8, 28.8, 22.6, 8.6; IR (film) $v_{\max } 3458,3400,3060,2982,2930,2854,1713,1668,1628,1607$, $1526,1367,1265,1238,1095,1082 \mathrm{~cm}^{-1}$; HRMS (ESI +$) \mathrm{m} / \mathrm{z} 546.2112\left(\mathrm{M}+\mathrm{H}^{+}, \mathrm{C}_{31} \mathrm{H}_{32} \mathrm{NO}_{8}\right.$ requires $\mathrm{m} / \mathrm{z}$ $546.2128)$.<smiles>CO[C@@H]1O[C@@H](Oc2ccc3cc(NC(=O)c4cccc(-c5ccccc5)c4)c(=O)oc3c2C)[C@@H]2OC(=O)O[C@H]12</smiles>

13a

$N$-(7-((3aR,4R,7R,7aR)-7-Methoxy-6,6-dimethyl-2-oxotetrahydro-3aH-[1,3]dioxolo[4,5-

c]pyran-4-yloxy)-8-methyl-2-oxo-2H-chromen-3-yl)biphenyl-3-carboxamide (13a). Colorless solid (58\%): $[\alpha]^{25}{ }_{\mathrm{D}}=-19.2^{\circ}\left(\mathrm{c}=0.12,20 \% \mathrm{MeOH}\right.$ in $\left.\mathrm{CH}_{2} \mathrm{Cl}_{2}\right) ;{ }^{1} \mathrm{H} \mathrm{NMR}\left(400 \mathrm{MHz}, \mathrm{CD}_{2} \mathrm{Cl}_{2}\right) \delta 8.86(\mathrm{~s}, 1 \mathrm{H})$, $8.83(\mathrm{~s}, 1 \mathrm{H}), 8.18(\mathrm{t}, J=1.7 \mathrm{~Hz}, 1 \mathrm{H}), 7.99-7.85(\mathrm{~m}, 2 \mathrm{H}), 7.71(\mathrm{dd}, J=1.4,8.5 \mathrm{~Hz}, 2 \mathrm{H}), 7.64(\mathrm{t}, J=7.8$ $\mathrm{Hz}, 1 \mathrm{H}), 7.54$ (t, $J=7.4 \mathrm{~Hz}, 2 \mathrm{H}), 7.46(\mathrm{dd}, J=1.7,7.4 \mathrm{~Hz}, 2 \mathrm{H}), 7.21(\mathrm{~d}, J=8.7 \mathrm{~Hz}, 1 \mathrm{H}), 5.86(\mathrm{~d}, J=2.2$ $\mathrm{Hz}, 1 \mathrm{H}), 5.11$ (dd, $J=2.2,7.8 \mathrm{~Hz}, 1 \mathrm{H}), 5.02$ (t, $J=7.8 \mathrm{~Hz}, 1 \mathrm{H}), 3.62(\mathrm{~s}, 3 \mathrm{H}), 3.39$ (d, $J=7.8 \mathrm{~Hz}, 1 \mathrm{H}), 2.35$ (s, 3H), 1.40 (s, 3H), 1.25 (s, 3H); ${ }^{13} \mathrm{C}$ NMR (100 MHz, $\left.\mathrm{CD}_{2} \mathrm{Cl}_{2}\right) \delta$ 166.2, 159.4, 155.6, 153.6, 149.6, $142.4,140.3$, 134.9, 131.4, 129.7, 129.3 (3C), 128.3, 127.6 (2C), 126.3, 126.2, 124.2, 122.8, 115.2, 115.1, 
111.7, 94.9, 83.2, 78.3, 77.5, 77.0, 60.8, 27.7, 22.4, 8.5; IR (film) $v_{\max } 3398,3063,2984,2934,1809,1713$, 1674, 1607, 1522, 1369, 1261, 1236, 1173, 1155, 1097, $1078 \mathrm{~cm}^{-1}$; HRMS (ESI +$) \mathrm{m} / \mathrm{z} 572.1901\left(\mathrm{M}+\mathrm{H}^{+}\right.$, $\mathrm{C}_{32} \mathrm{H}_{30} \mathrm{NO}_{9}$ requires $m / z$ 572.1921).

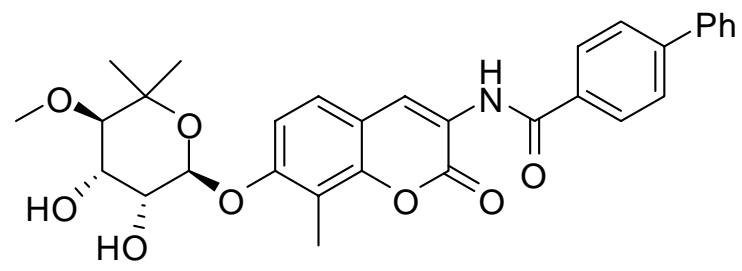

14

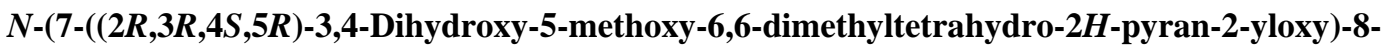
methyl-2-oxo-2H-chromen-3-yl)biphenyl-4-carboxamide (14). Colorless solid (80\%): $[\alpha]_{\mathrm{D}}^{26}=-7.3^{\circ}(\mathrm{c}$ $=0.06,20 \% \mathrm{MeOH}$ in $\left.\mathrm{CH}_{2} \mathrm{Cl}_{2}\right) ;{ }^{1} \mathrm{H} \mathrm{NMR}\left(400 \mathrm{MHz}, 20 \% \mathrm{CD}_{3} \mathrm{OD}\right.$ in $\left.\mathrm{CD}_{2} \mathrm{Cl}_{2}\right) \delta 8.87(\mathrm{~s}, 1 \mathrm{H}), 8.78(\mathrm{~s}, 1 \mathrm{H})$, $7.98(\mathrm{~d}, J=8.4 \mathrm{~Hz}, 2 \mathrm{H}), 7.75(\mathrm{~d}, J=8.4 \mathrm{~Hz}, 2 \mathrm{H}), 7.65(\mathrm{dd}, J=1.3,7.8 \mathrm{~Hz}, 2 \mathrm{H}), 7.47(\mathrm{t}, J=7.4 \mathrm{~Hz}, 2 \mathrm{H})$, $7.38(\mathrm{~d}, J=7.8 \mathrm{~Hz}, 2 \mathrm{H}), 7.21(\mathrm{~d}, J=8.4 \mathrm{~Hz}, 1 \mathrm{H}), 5.55(\mathrm{~d}, J=2.1 \mathrm{~Hz}, 1 \mathrm{H}), 4.18-4.08(\mathrm{~m}, 2 \mathrm{H}), 3.80(\mathrm{~s}$, $3 \mathrm{H}), 3.35-3.30(\mathrm{~m}, 1 \mathrm{H}), 2.27(\mathrm{~s}, 3 \mathrm{H}), 1.32(\mathrm{~s}, 3 \mathrm{H}), 1.07(\mathrm{~s}, 3 \mathrm{H}) ;{ }^{13} \mathrm{C} \mathrm{NMR}\left(100 \mathrm{MHz}, 20 \% \mathrm{CD}_{3} \mathrm{OD}\right.$ in $\left.\mathrm{CD}_{2} \mathrm{Cl}_{2}\right) \delta 159.9,156.7,148.9,145.8,138.7,129.3(2 \mathrm{C}), 128.6,128.1(3 \mathrm{C}), 127.8(2 \mathrm{C}), 127.5(3 \mathrm{C}), 126.2$, 125.3, 122.8, 114.5, 114.2, 111.6, 98.8, 84.5, 78.9, 71.6, 68.8, 61.9, 28.9, 22.6, 8.3; IR (film) $v_{\max } 3404$, 3059, 3032, 2978, 2932, 2835, 1709, 1666, 1609, 1531, 1416, 1367, 1265, 1252, 1095, $1078 \mathrm{~cm}^{-1}$; HRMS (ESI+) $m / z 546.2140\left(\mathrm{M}+\mathrm{H}^{+}, \mathrm{C}_{31} \mathrm{H}_{32} \mathrm{NO}_{8}\right.$ requires $m / z$ 546.2128).<smiles>CO[C@@H]1O[C@@H](Oc2ccc3cc(NC(=O)c4ccc(-c5ccccc5)cc4)c(=O)oc3c2C)[C@@H]2OC(=O)O[C@H]12</smiles>

$14 a$

N-(7-((3aR,4R,7R,7aR)-7-Methoxy-6,6-dimethyl-2-oxotetrahydro-3aH-[1,3]dioxolo[4,5-

c]pyran-4-yloxy)-8-methyl-2-oxo-2H-chromen-3-yl)biphenyl-4-carboxamide (14a). Colorless solid $(32 \%):[\alpha]^{25}=-17.3^{\circ}\left(\mathrm{c}=0.08,20 \% \mathrm{MeOH}\right.$ in $\left.\mathrm{CH}_{2} \mathrm{Cl}_{2}\right) ;{ }^{1} \mathrm{H} \mathrm{NMR}\left(400 \mathrm{MHz}, \mathrm{CDCl}_{3}\right) \delta 8.86(\mathrm{~s}, 1 \mathrm{H})$, $8.83(\mathrm{~s}, 1 \mathrm{H}), 8.02(\mathrm{~d}, J=8.4 \mathrm{~Hz}, 2 \mathrm{H}), 7.76(\mathrm{~d}, J=8.4 \mathrm{~Hz}, 2 \mathrm{H}), 7.67(\mathrm{dd}, J=1.3,7.8 \mathrm{~Hz}, 2 \mathrm{H}), 7.51(\mathrm{t}, J=$ $7.8 \mathrm{~Hz}, 2 \mathrm{H}), 7.44$ (d, $J=7.3 \mathrm{~Hz}, 1 \mathrm{H}), 7.41(\mathrm{~d}, J=8.4 \mathrm{~Hz}, 1 \mathrm{H}), 7.18(\mathrm{~d}, J=8.4 \mathrm{~Hz}, 1 \mathrm{H}), 5.82(\mathrm{~d}, J=1.8 \mathrm{~Hz}$, 1H), 5.09 (dd, $J=1.8,7.9 \mathrm{~Hz}, 1 \mathrm{H}), 4.99$ (t, $J=7.9 \mathrm{~Hz}, 1 \mathrm{H}), 3.62$ (s, 3H), 3.34 (d, J= 7.6 Hz, 1H), 2.39 (s, $3 \mathrm{H}), 1.39(\mathrm{~s}, 3 \mathrm{H}), 1.23(\mathrm{~s}, 3 \mathrm{H}) ;{ }^{13} \mathrm{C} \mathrm{NMR}\left(100 \mathrm{MHz}, \mathrm{CDCl}_{3}\right) \delta 165.8,159.5,155.6,153.6,149.5,145.4$, 140.0, 132.8, 129.4 (2C), 128.8, 128.1 (2C), 127.8 (2C), 127.2 (2C), 126.2, 124.1, 122.8, 115.2, 115.1, 111.7, 94.8, 83.2, 78.3, 77.5, 77.0, 60.8, 27.7, 22.4, 8.6; IR (film) $v_{\max } 3400,3032,2986,2935,2851,1811$, 
1713, 1672, 1609, 1529, 1512, 1367, 1248, 1173, $1095 \mathrm{~cm}^{-1}$; HRMS (ESI +$) \mathrm{m} / \mathrm{z} 572.1924\left(\mathrm{M}+\mathrm{H}^{+}\right.$, $\mathrm{C}_{32} \mathrm{H}_{30} \mathrm{NO}_{9}$ requires $m / z$ 572.1921).<smiles>CO[C@H]1[C@@H](O)[C@@H](O)C(Oc2ccc3cc(NC(=O)c4ccccc4[N+](=O)[O-])c(=O)oc3c2C)OC1(C)C</smiles>

15

$N$-(7-((2R,3R,4S,5R)-3,4-Dihydroxy-5-methoxy-6,6-dimethyltetrahydro-2H-pyran-2-yloxy)-8methyl-2-oxo-2H-chromen-3-yl)-2-nitrobenzamide (15). Prepared as described above with the exception that the product was purified by preparative TLC $\left(\mathrm{SiO}_{2}, 1: 1\right.$ hexanes:ethyl acetate, developed 5 times $)$ to afford $15(44 \%)$ as a yellow solid: $[\alpha]^{23}{ }_{\mathrm{D}}=-15.9^{\circ}\left(\mathrm{c}=0.15,20 \% \mathrm{MeOH}\right.$ in $\left.\mathrm{CH}_{2} \mathrm{Cl}_{2}\right) ;{ }^{1} \mathrm{H} \mathrm{NMR}(400 \mathrm{MHz}$, $20 \% \mathrm{CD}_{3} \mathrm{OD}$ in $\left.\mathrm{CD}_{2} \mathrm{Cl}_{2}\right) \delta 8.75(\mathrm{~s}, 1 \mathrm{H}), 8.15(\mathrm{dd}, J=0.9,8.1 \mathrm{~Hz}, 1 \mathrm{H}), 7.79-7.61(\mathrm{~m}, 3 \mathrm{H}), 7.37(\mathrm{~d}, J=8.8$ $\mathrm{Hz}, 1 \mathrm{H}), 7.20(\mathrm{~d}, J=8.8 \mathrm{~Hz}, 1 \mathrm{H}), 5.56(\mathrm{~d}, J=2.0 \mathrm{~Hz}, 1 \mathrm{H}), 4.19-4.08(\mathrm{~m}, 2 \mathrm{H}), 3.59$ (s, 3H), 3.35 (d, $J=$ $9.1 \mathrm{~Hz}, 1 \mathrm{H}), 2.25$ (s, 3H), 1.34 (s, 3H), 1.09 (s, 3H); ${ }^{13} \mathrm{C} \mathrm{NMR}\left(125 \mathrm{MHz}, \mathrm{CD}_{2} \mathrm{Cl}_{2}\right) \delta 165.1,159.2,156.8$, 149.8, 146.9, 134.4, 132.2, 131.8, 128.9, 126.4, 125.6, 125.2, 122.0, 114.6, 114.0, 111.6, 98.2, 84.6, 78.9, 71.6, 69.0, 62.1, 29.3, 22.6, 8.5; IR (film) $v_{\max } 3441,3387,2984,2934,1713,1674,1607,1529,1371$, $1348,1256,1105,1084 \mathrm{~cm}^{-1}$; HRMS (ESI+) $\mathrm{m} / \mathrm{z} 515.1669\left(\mathrm{M}+\mathrm{H}^{+}, \mathrm{C}_{25} \mathrm{H}_{27} \mathrm{~N}_{2} \mathrm{O}_{10}\right.$ requires $\mathrm{m} / \mathrm{z}$ 515.1666).

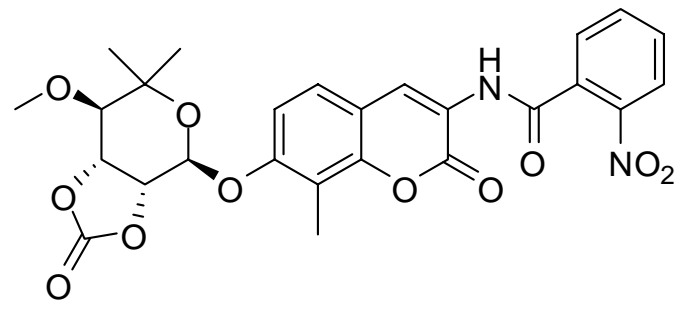

\section{5a}

\section{$N-(7-((3 a R, 4 R, 7 R, 7 a R)-7-M e t h o x y-6,6-d i m e t h y l-2-o x o t e t r a h y d r o-3 a H-[1,3]$ dioxolo[4,5-}

c]pyran-4-yloxy)-8-methyl-2-oxo-2H-chromen-3-yl)-2-nitrobenzamide (15a). Yellow solid (74\%): $[\alpha]^{26}=-19.5^{\circ}\left(\mathrm{c}=0.55,20 \% \mathrm{MeOH}\right.$ in $\left.\mathrm{CH}_{2} \mathrm{Cl}_{2}\right) ;{ }^{1} \mathrm{H} \mathrm{NMR}\left(400 \mathrm{MHz}, \mathrm{CDCl}_{3}\right) \delta 8.79(\mathrm{~s}, 1 \mathrm{H}), 8.41(\mathrm{~s}$, 1H), $8.17(\mathrm{dd}, J=0.9,8.1 \mathrm{~Hz}, 1 \mathrm{H}), 7.80-7.64(\mathrm{~m}, 3 \mathrm{H}), 7.41(\mathrm{~d}, J=8.7 \mathrm{~Hz}, 1 \mathrm{H}), 7.17$ (d, $J=8.7 \mathrm{~Hz}, 1 \mathrm{H})$, $5.82(\mathrm{~d}, J=1.8 \mathrm{~Hz}, 1 \mathrm{H}), 5.09(\mathrm{dd}, J=1.8,7.9 \mathrm{~Hz}, 1 \mathrm{H}), 4.98(\mathrm{t}, J=7.9 \mathrm{~Hz}, 1 \mathrm{H}), 3.61(\mathrm{~s}, 3 \mathrm{H}), 3.34(\mathrm{~d}, J=$ $7.9 \mathrm{~Hz}, 1 \mathrm{H}), 2.32$ (s, 3H), $1.38(\mathrm{~s}, 3 \mathrm{H}), 1.13(\mathrm{~s}, 3 \mathrm{H}) ;{ }^{13} \mathrm{C} \mathrm{NMR}\left(100 \mathrm{MHz}, \mathrm{CDCl}_{3}\right) \delta 165.4,159.2,155.9$, 153.6, 149.7, 146.8, 134.4, 132.2, 131.7, 128.8, 126.5, 125.3, 122.2, 115.3, 114.8, 111.8, 94.7, 83.3, 78.3, $77.7,77.5,77.1,61.0,27.9,22.6,8.8$; IR (film) $v_{\max } 3379,3310,3088,2986,2937,2885,2841,1809$, 
1713, 1676, 1607, 1529, 1371, 1348, 1252, 1171, 1105, 1086, 1072, 1036, $1003 \mathrm{~cm}^{-1}$; HRMS (ESI+) $\mathrm{m} / \mathrm{z}$ $541.1441\left(\mathrm{M}+\mathrm{H}^{+}, \mathrm{C}_{26} \mathrm{H}_{25} \mathrm{~N}_{2} \mathrm{O}_{11}\right.$ requires $m / z$ 541.1458).<smiles>COC1[C@H](O)[C@@H](O)C(Oc2ccc3cc(NC(=O)c4cccc([N+](=O)[O-])c4)c(=O)oc3c2C)OC1(C)C</smiles>

16

$N$-(7-((2R,3R,4S,5R)-3,4-Dihydroxy-5-methoxy-6,6-dimethyltetrahydro-2H-pyran-2-yloxy)-8methyl-2-oxo-2H-chromen-3-yl)-3-nitrobenzamide (16). Yellow solid $(73 \%):[\alpha]_{D}^{25}=-15.7^{\circ}(\mathrm{c}=0.26$, $20 \% \mathrm{MeOH}$ in $\left.\mathrm{CH}_{2} \mathrm{Cl}_{2}\right) ;{ }^{1} \mathrm{H}$ NMR $\left(400 \mathrm{MHz}, \mathrm{CDCl}_{3}\right) \delta 8.71(\mathrm{~s}, 2 \mathrm{H}), 8.36(\mathrm{~d}, J=1.0,8.2 \mathrm{~Hz}, 1 \mathrm{H}) 8.20(\mathrm{~d}, J$ $=1.0,8.2 \mathrm{~Hz}, 1 \mathrm{H}), 7.68(\mathrm{t}, J=8.0 \mathrm{~Hz}, 1 \mathrm{H}), 7.31(\mathrm{~d}, J=8.7 \mathrm{~Hz}, 1 \mathrm{H}), 7.15(\mathrm{~d}, J=8.7 \mathrm{~Hz}, 1 \mathrm{H}), 5.51(\mathrm{~d}, J=$ $2.0 \mathrm{~Hz}, 1 \mathrm{H}), 4.14-4.05$ (m, 2H), 3.59 (s, 3H), 3.31 (d, J=9.1 Hz, 1H), 2.33 (s, 3H), 1.29 (s, 3H), 1.10 (s, $3 \mathrm{H}) ;{ }^{13} \mathrm{C} \mathrm{NMR}\left(100 \mathrm{MHz}, \mathrm{CDCl}_{3}\right) \delta 164.3,159.7,157.0,149.7,148.8,135.7,133.2,130.5,127.1,126.7$, $126.4,122.9,121.4,114.6,113.8,111.7,98.7,84.5,79.0,71.5,68.8,62.1,29.2,22.7,8.5$; IR (film) $v_{\max }$ 3362, 2986, 2949, 2837, 1705, 1645, 1635, 1605, 1554, 1531, 1371, 1346, 1253, 1136, 1117, 1003, 1080, $1018 \mathrm{~cm}^{-1}$; HRMS (ESI+) m/z $537.1477\left(\mathrm{M}+\mathrm{Na}^{+}, \mathrm{C}_{25} \mathrm{H}_{26} \mathrm{~N}_{2} \mathrm{O}_{10} \mathrm{Na}\right.$ requires $\mathrm{m} / \mathrm{z}$ 537.1485).<smiles>CO[C@H]1[C@H]2OC(=O)O[C@H]2[C@H](Oc2ccc3cc(NC(=O)c4cccc([N+](=O)[O-])c4)c(=O)oc3c2C)OC1(C)C</smiles>

16a

$N$-(7-((3aR,4R,7R,7aR)-7-Methoxy-6,6-dimethyl-2-oxotetrahydro-3aH-[1,3]dioxolo[4,5-

c]pyran-4-yloxy)-8-methyl-2-oxo-2H-chromen-3-yl)-3-nitrobenzamide (16a). Yellow solid (71\%): $[\alpha]^{26}{ }_{\mathrm{D}}=-28.4^{\circ}\left(\mathrm{c}=0.29,20 \% \mathrm{MeOH}\right.$ in $\left.\mathrm{CH}_{2} \mathrm{Cl}_{2}\right) ;{ }^{1} \mathrm{H}$ NMR $\left(400 \mathrm{MHz}, \mathrm{CD}_{2} \mathrm{Cl}_{2}\right) \delta 8.83(\mathrm{~s}, 1 \mathrm{H}), 8.82(\mathrm{~s}$, 1H) $8.77(\mathrm{t}, J=1.9 \mathrm{~Hz}, 1 \mathrm{H}), 8.46(\mathrm{td}, J=1.9,8.2 \mathrm{~Hz}, 1 \mathrm{H}), 8.27$ (d, $J=8.2 \mathrm{~Hz}, 1 \mathrm{H}), 7.78$ (t, $J=8.2 \mathrm{~Hz}$, 1H), $7.46(\mathrm{~d}, J=8.7 \mathrm{~Hz}, 1 \mathrm{H}), 7.21(\mathrm{~d}, J=8.7 \mathrm{~Hz}, 1 \mathrm{H}), 5.86(\mathrm{~d}, J=2.1 \mathrm{~Hz}, 1 \mathrm{H}), 5.12(\mathrm{dd}, J=2.1,8.0 \mathrm{~Hz}$, 1H), 5.03 (t, $J=8.0 \mathrm{~Hz}, 1 \mathrm{H}), 3.62(\mathrm{~s}, 3 \mathrm{H}), 3.39$ (d, $J=8.0 \mathrm{~Hz}, 1 \mathrm{H}), 2.33$ (s, 3H), 1.40 (s, 3H), 1.18 (s, 3H); ${ }^{13} \mathrm{C}$ NMR $\left(100 \mathrm{MHz}, \mathrm{CD}_{2} \mathrm{Cl}_{2}\right) \delta 163.9,159.3,155.9,153.6,149.7,148.9,135.8,133.1,130.6,127.1,126.4$, $125.1,122.8,122.2,115.3,114.8,111.7,94.8,83.2,78.3,77.4,77.0,60.8,27.7,22.4,8.6$; IR (film) $v_{\max }$ 3516, 3389, 3088, 3065, 2986, 2939, 2837, 1809, 1713, 1674, 1607, 1529, 1371, 1350, 1249, 1173, 1109 , 1090, $1036 \mathrm{~cm}^{-1}$; HRMS (ESI+) m/z 563.1249 (M + Na ${ }^{+}, \mathrm{C}_{26} \mathrm{H}_{24} \mathrm{~N}_{2} \mathrm{O}_{11} \mathrm{Na}$ requires $\left.\mathrm{m} / \mathrm{z} 563.1278\right)$. 


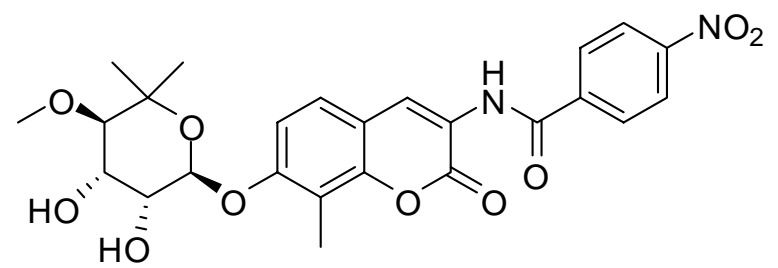

17

N-(7-((2R,3R,4S,5R)-3,4-Dihydroxy-5-methoxy-6,6-dimethyltetrahydro-2H-pyran-2-yloxy)-8methyl-2-oxo-2H-chromen-3-yl)-4-nitrobenzamide (17). Yellow solid (79\%): $[\alpha]_{\mathrm{D}}^{26}=-13.1^{\mathrm{o}}(\mathrm{c}=0.16$, 20\% $\mathrm{MeOH}$ in $\left.\mathrm{CH}_{2} \mathrm{Cl}_{2}\right) ;{ }^{1} \mathrm{H}$ NMR $\left(400 \mathrm{MHz}, 20 \% \mathrm{CD}_{3} \mathrm{OD}\right.$ in $\left.\mathrm{CD}_{2} \mathrm{Cl}_{2}\right) \delta 9.18(\mathrm{~s}, 1 \mathrm{H}), 8.79(\mathrm{dd}, J=1.9,6.9$ $\mathrm{Hz}, 2 \mathrm{H}) 8.56(\mathrm{dd}, J=1.9,6.9 \mathrm{~Hz}, 2 \mathrm{H}), 7.85(\mathrm{~d}, J=8.7 \mathrm{~Hz}, 1 \mathrm{H}), 7.67$ (d, $J=8.7 \mathrm{~Hz}, 1 \mathrm{H}), 6.00(\mathrm{~d}, J=2.2$ $\mathrm{Hz}, 1 \mathrm{H}), 4.60(\mathrm{dd}, J=3.4,9.4 \mathrm{~Hz}, 1 \mathrm{H}), 4.56(\mathrm{t}, J=3.4 \mathrm{~Hz}, 1 \mathrm{H}), 4.02(\mathrm{~s}, 3 \mathrm{H}), 3.80(\mathrm{~d}, J=9.4 \mathrm{~Hz}, 1 \mathrm{H}), 2.71$ $(\mathrm{s}, 3 \mathrm{H}), 1.75(\mathrm{~s}, 3 \mathrm{H}), 1.61(\mathrm{~s}, 3 \mathrm{H}) ;{ }^{13} \mathrm{C} \mathrm{NMR}\left(100 \mathrm{MHz}, 20 \% \mathrm{CD}_{3} \mathrm{OD}\right.$ in $\left.\mathrm{CD}_{2} \mathrm{Cl}_{2}\right) \delta 165.0,159.6,157.1$, 150.3, 149.9, 139.7, 129.0 (2C), 127.0, 126.4, 124.2 (2C), 121.5, 114.5, 113.8, 111.7, 98.9, 84.4, 79.0, 71.6, 68.7, 61.8, 28.8, 22.5, 8.1; IR (film) $v_{\max } 3381,3053,2947,2835,1699,1666,1603,1524,1373,1346$, 1252, 1113, 1086, $1018 \mathrm{~cm}^{-1}$; HRMS (ESI+) $\mathrm{m} / \mathrm{z} 537.1486\left(\mathrm{M}+\mathrm{Na}^{+}, \mathrm{C}_{25} \mathrm{H}_{26} \mathrm{~N}_{2} \mathrm{O}_{10} \mathrm{Na}\right.$ requires $\mathrm{m} / \mathrm{z}$ $537.1485)$.

General procedure for reduction of nitro group: Palladium on carbon $(10 \%, 0.1$ eq) was added to a solution of 15, 16 or 17 ( 1 eq) in THF at rt. The suspension was stirred for $6 \mathrm{~h}$ under a hydrogen atmosphere, filtered through a plug of $\mathrm{SiO}_{2}$, and eluted with THF. The eluent was concentrated and the residue purified by preparative $\mathrm{TLC}\left(\mathrm{SiO}_{2}, 100: 1 \rightarrow 50: 1 ; \mathrm{CH}_{2} \mathrm{Cl}_{2}\right.$ :acetone $)$ to afford the aniline.

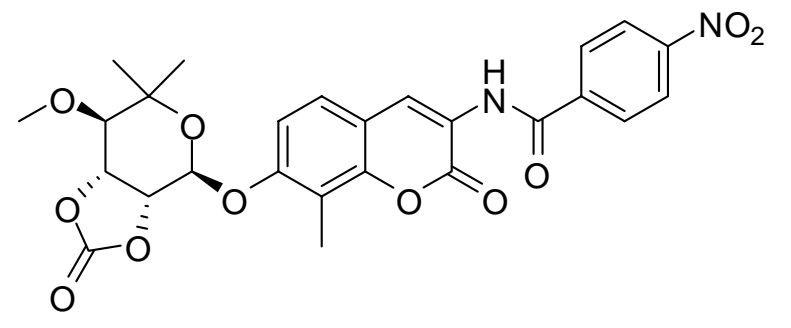

17a

$N-(7-((3 a R, 4 R, 7 R, 7 a R)-7-M e t h o x y-6,6-d i m e t h y l-2-o x o t e t r a h y d r o-3 a H-[1,3] d i o x o l o[4,5-$

c]pyran-4-yloxy)-8-methyl-2-oxo-2H-chromen-3-yl)-4-nitrobenzamide (17a). Yellow solid (95\%): $[\alpha]^{24}=-29.5^{\circ}\left(\mathrm{c}=0.20,20 \% \mathrm{MeOH}\right.$ in $\left.\mathrm{CH}_{2} \mathrm{Cl}_{2}\right) ;{ }^{1} \mathrm{H} \mathrm{NMR}\left(400 \mathrm{MHz}, \mathrm{CD}_{2} \mathrm{Cl}_{2}\right) \delta 8.83(\mathrm{~d}, J=2.2 \mathrm{~Hz}, 2 \mathrm{H})$, $8.39(\mathrm{dd}, J=1.7,8.3 \mathrm{~Hz}, 2 \mathrm{H}) 8.13(\mathrm{dt}, J=2.2,8.3 \mathrm{~Hz}, 2 \mathrm{H}), 7.47$ (d, $J=8.8 \mathrm{~Hz}, 1 \mathrm{H}), 7.22(\mathrm{~d}, J=8.8 \mathrm{~Hz}$, $1 \mathrm{H}), 5.86(\mathrm{~d}, J=2.1 \mathrm{~Hz}, 1 \mathrm{H}), 5.12(\mathrm{dd}, J=2.1,7.8 \mathrm{~Hz}, 1 \mathrm{H}), 5.03(\mathrm{t}, J=7.8 \mathrm{~Hz}, 1 \mathrm{H}), 3.62(\mathrm{~s}, 3 \mathrm{H}), 3.39$ (d, $J=7.8 \mathrm{~Hz}, 1 \mathrm{H}), 2.34(\mathrm{~s}, 3 \mathrm{H}), 1.40(\mathrm{~s}, 3 \mathrm{H}), 1.24(\mathrm{~s}, 3 \mathrm{H}) ;{ }^{13} \mathrm{C} \mathrm{NMR}\left(100 \mathrm{MHz}, \mathrm{CD}_{2} \mathrm{Cl}_{2}\right) \delta 164.3,159.3$, 155.9, 153.6, 150.4, 149.7, 139.6, 128.8 (2C), 126.4, 125.1, 122.4 (2C), 122.2, 115.3, 114.8, 111.7, 94.8, 83.2, 78.3, 77.5, 77.0, 60.8, 27.7, 22.4, 8.5; IR (film) $v_{\max } 3383,3364,3105,2982,2945,2833,1811,1709$, 
1672, 1605, 1529, 1371, 1346, 1177, 1109, 1092, $1028 \mathrm{~cm}^{-1} ;$ HRMS (ESI+) m/z $563.1273\left(\mathrm{M}^{+} \mathrm{Na}^{+}\right.$, $\mathrm{C}_{26} \mathrm{H}_{24} \mathrm{~N}_{2} \mathrm{O}_{11} \mathrm{Na}$ requires $m / z$ 563.1278).

General procedure for solvolysis of the cyclic carbonate: $\mathrm{Et}_{3} \mathrm{~N}(10 \%$ total volume) was added dropwise to a solution of cyclic carbonate in methanol. The resulting mixture was stirred for $14 \mathrm{~h}$, and then concentrated. The residue was purified via preparative TLC or column chromtography $\left(\mathrm{SiO}_{2}, 4: 1\right.$; $\mathrm{CH}_{2} \mathrm{Cl}_{2}$ :acetone).<smiles>CO[C@H]1[C@@H](O)[C@@H](O)C(Oc2ccc3cc(NC(=O)c4ccccc4N)c(=O)oc3c2C)OC1(C)C</smiles>

18

2-Amino- $N$-(7-((2R,3R,4S,5R)-3,4-dihydroxy-5-methoxy-6,6-dimethyltetrahydro-2H-pyran2-yloxy)-8-methyl-2-oxo-2H-chromen-3-yl)benzamide (18). Colorless solid $(90 \%)$ : $[\alpha]_{\mathrm{D}}^{23}=-17.6^{\mathrm{o}}(\mathrm{c}=$ 0.09, 20\% $\mathrm{MeOH}$ in $\left.\mathrm{CH}_{2} \mathrm{Cl}_{2}\right) ;{ }^{1} \mathrm{H} \mathrm{NMR}\left(500 \mathrm{MHz}, 20 \% \mathrm{CD}_{3} \mathrm{OD}\right.$ in $\left.\mathrm{CD}_{2} \mathrm{Cl}_{2}\right) \delta 8.64(\mathrm{~s}, 1 \mathrm{H}), 7.48(\mathrm{dd}, J=$ 1.0, $7.5 \mathrm{~Hz}, 1 \mathrm{H}), 7.31(\mathrm{~d}, J=9.0 \mathrm{~Hz}, 1 \mathrm{H}), 7.24-7.17(\mathrm{~m}, 1 \mathrm{H}), 7.14(\mathrm{~d}, J=8.5 \mathrm{~Hz}, 1 \mathrm{H}), 6.70(\mathrm{~d}, J=8.5$ $\mathrm{Hz}, 1 \mathrm{H}), 6.66(\mathrm{t}, J=7.5 \mathrm{~Hz}, 1 \mathrm{H}), 5.48(\mathrm{~d}, J=2.0 \mathrm{~Hz}, 1 \mathrm{H}), 4.09(\mathrm{dd}, J=3.3,9.5 \mathrm{~Hz}, 1 \mathrm{H}), 4.04(\mathrm{t}, J=3.3$ $\mathrm{Hz}, 1 \mathrm{H}), 3.50(\mathrm{~s}, 3 \mathrm{H}), 3.28-3.25(\mathrm{~m}, 1 \mathrm{H}), 2.21(\mathrm{~s}, 3 \mathrm{H}), 1.26(\mathrm{~s}, 3 \mathrm{H}), 1.03(\mathrm{~s}, 3 \mathrm{H}) ;{ }^{13} \mathrm{C} \mathrm{NMR}(125 \mathrm{MHz}$, $20 \% \mathrm{CD}_{3} \mathrm{OD}$ in $\left.\mathrm{CD}_{2} \mathrm{Cl}_{2}\right) \delta 168.4,159.9,156.5,149.6,149.5,133.6,127.9,126.0,124.6,122.1,118.0$, 117.2, 115.3, 114.5, 114.3, 111.6, 98.8, 84.5, 78.9, 71.6, 68.8, 61.9, 28.9, 22.6, 8.3; IR (film) $v_{\max } 3470$, 3408, 3362, 2978, 2926, 2853, 1707, 1657, 1609, 1520, 1450, 1408, 1367, 1263, $12421088 \mathrm{~cm}^{-1}$; HRMS (ESI+) $m / z 485.1919\left(\mathrm{M}+\mathrm{H}^{+}, \mathrm{C}_{25} \mathrm{H}_{29} \mathrm{~N}_{2} \mathrm{O}_{8}\right.$ requires $m / z$ 485.1924).<smiles>CO[C@H]1[C@@H](O)[C@@H](O)C(Oc2ccc3cc(NC(=O)c4cccc(N)c4)c(=O)oc3c2C)OC1(C)C</smiles>

19

3-Amino- $N$-(7-((2R,3R,4S,5R)-3,4-dihydroxy-5-methoxy-6,6-dimethyltetrahydro-2H-pyran2-yloxy)-8-methyl-2-oxo-2H-chromen-3-yl)benzamide (19). Colorless solid $(77 \%):[\alpha]^{26}=-24.3^{\circ}(\mathrm{c}=$ 0.07, 20\% $\mathrm{MeOH}$ in $\left.\mathrm{CH}_{2} \mathrm{Cl}_{2}\right) ;{ }^{1} \mathrm{H} \mathrm{NMR}\left(400 \mathrm{MHz}, 20 \% \mathrm{CD}_{3} \mathrm{OD}\right.$ in $\left.\mathrm{CD}_{2} \mathrm{Cl}_{2}\right) \delta 8.79(\mathrm{~s}, 1 \mathrm{H}), 8.76(\mathrm{~s}, 1 \mathrm{H})$, $7.41(\mathrm{~d}, J=8.7 \mathrm{~Hz}, 1 \mathrm{H}), 7.32-7.20(\mathrm{~m}, 4 \mathrm{H}), 6.92(\mathrm{~d}, J=6.3 \mathrm{~Hz}, 1 \mathrm{H}), 5.58(\mathrm{~d}, J=1.6 \mathrm{~Hz}, 1 \mathrm{H}), 4.20-4.11$ $(\mathrm{m}, 2 \mathrm{H}), 3.60(\mathrm{~s}, 3 \mathrm{H}), 3.35(\mathrm{~d}, J=9.5 \mathrm{~Hz}, 1 \mathrm{H}), 2.30(\mathrm{~s}, 3 \mathrm{H}), 1.35(\mathrm{~s}, 3 \mathrm{H}), 1.17(\mathrm{~s}, 3 \mathrm{H}) ;{ }^{13} \mathrm{C} \mathrm{NMR}(200$ 
$\mathrm{MHz}, 20 \% \mathrm{CD}_{3} \mathrm{OD}$ in $\left.\mathrm{CD}_{2} \mathrm{Cl}_{2}\right) \delta 166.8,159.4,156.3,149.2,147.7,134.7,129.6,125.7,124.8,121.6$, 118.9, 116.3, 114.1, 113.8, 113.2, 111.2, 98.4, 84.1, 78.5, 71.2, 68.4, 61.4, 28.5, 22.2, 7.8; IR (film) $v_{\max }$ 3404, 2986, 2949, 2843, 1634, 1607, 1520, 1367, 1261, 1111, $1016 \mathrm{~cm}^{-1}$; HRMS (ESI+) m/z 507.1740 (M $+\mathrm{Na}^{+}, \mathrm{C}_{25} \mathrm{H}_{28} \mathrm{~N}_{2} \mathrm{O}_{8} \mathrm{Na}$ requires $\mathrm{m} / \mathrm{z}$ 507.1743).<smiles>CO[C@H]1[C@@H](O)[C@@H](O)[C@H](Oc2ccc3cc(NC(=O)c4ccc(N)cc4)c(=O)oc3c2C)OC1(C)C</smiles>

20

4-Amino- $N$-(7-((2R,3R,4S,5R)-3,4-dihydroxy-5-methoxy-6,6-dimethyltetrahydro-2H-pyran2-yloxy)-8-methyl-2-oxo-2H-chromen-3-yl)benzamide (20). Yellow solid (77\%): $[\alpha]^{26}{ }_{\mathrm{D}}=-15.9^{\circ}(\mathrm{c}=$ $0.30,20 \% \mathrm{MeOH}$ in $\mathrm{CH}_{2} \mathrm{Cl}_{2}$ ); ${ }^{1} \mathrm{H} \mathrm{NMR}\left(400 \mathrm{MHz}, \mathrm{CDCl}_{3}\right) \delta 8.76(\mathrm{~s}, 1 \mathrm{H}), 8.61(\mathrm{~s}, 1 \mathrm{H}), 7.75$ (dd, $J=1.8$, $6.8 \mathrm{~Hz}, 2 \mathrm{H}), 7.31(\mathrm{~d}, J=8.7 \mathrm{~Hz}, 1 \mathrm{H}), 7.17(\mathrm{~d}, J=8.7 \mathrm{~Hz}, 1 \mathrm{H}), 6.73(\mathrm{td}, J=1.8,6.8 \mathrm{~Hz}, 2 \mathrm{H}), 5.60(\mathrm{~d}, J=$ $1.5 \mathrm{~Hz}, 1 \mathrm{H}), 4.28-4.24$ (m, 2H), 4.16 (s, 2H), 3.62 (s, 3H), 3.39 (d, J = 8.8 Hz, 1H), 3.17 (s, 1H), 2.90 (s, 1H), 2.27 (s, 3H), 1.39 (s, 3H), 1.14 (s, 3H); $\left.{ }^{13} \mathrm{C} \mathrm{NMR} \mathrm{(100} \mathrm{MHz,} \mathrm{CDCl}_{3}\right) \delta 166.2,159.9,156.7,150.9$, 149.3, 129.5 (2C), 126.1, 124.2, 123.3, 122.4, 114.7 (2C), 114.6 (2C), 111.5, 98.3, 84.7, 79.0, 71.6, 69.0, 62.3, 29.6, 22.9, 8.8; IR (film) $v_{\max } 3381,2980,2941,2839,1697,1634,1607,1531,1510,1367,1252$, 1184, 1092, 1078, 1020, 993, $966 \mathrm{~cm}^{-1}$; HRMS (ESI+) $\mathrm{m} / \mathrm{z} 485.1940\left(\mathrm{M}+\mathrm{H}^{+}, \mathrm{C}_{25} \mathrm{H}_{29} \mathrm{~N}_{2} \mathrm{O}_{8}\right.$ requires $\mathrm{m} / \mathrm{z}$ 485.1924).<smiles>CO[C@H]1[C@@H](O)[C@@H](O)C(Oc2ccc3cc(NS(=O)(=O)c4ccccc4)c(=O)oc3c2C)OC1(C)C</smiles>

21

$N$-(7-((2R,3R,4S,5R)-3,4-Dihydroxy-5-methoxy-6,6-dimethyltetrahydro-2H-pyran-2-yloxy)-8methyl-2-oxo-2H-chromen-3-yl)benzenesulfonamide (21). Benzenesulfonyl chloride (8 $\mu \mathrm{L}, 10.7 \mathrm{mg}$, $60.2 \mu \mathrm{mol})$ was added to a solution of $21.4 \mathrm{mg}(54.7 \mu \mathrm{mol})$ aniline $7 \mathrm{in} 0.5 \mathrm{~mL}$ of pyridine at $\mathrm{rt}$. The reaction mixture was stirred for $14 \mathrm{~h}$ and then concentrated. The residue was purified via preparative TLC (40:1 $\mathrm{CH}_{2} \mathrm{Cl}_{2}$ :acetone) to afford $20 \mathrm{mg}(69 \%)$ of the sulfonamide as a glassy solid. The cyclic carbonate $\left(20 \mathrm{mg}\right.$ ) was dissolved in $0.5 \mathrm{~mL}$ of methanol and $0.1 \mathrm{~mL}$ of $\mathrm{Et}_{3} \mathrm{~N}$ was added dropwise. The reaction mixture was stirred for $14 \mathrm{~h}$ at $\mathrm{rt}$ before concentration. The residue was purified by preparative TLC (10:1; $\mathrm{CH}_{2} \mathrm{Cl}_{2}$ :methanol) to afford $15 \mathrm{mg}(79 \%)$ of 21 as a white solid. $[\alpha]^{24}=-5.1^{\circ}(\mathrm{c}=0.42,20 \% \mathrm{MeOH}$ in $\mathrm{CH}_{2} \mathrm{Cl}_{2}$ ); ${ }^{1} \mathrm{H}$ NMR (400 MHz, $\mathrm{CD}_{2} \mathrm{Cl}_{2}$ ) $\delta 7.88(\mathrm{dd}, J=0.8,8.0 \mathrm{~Hz}, 2 \mathrm{H}), 7.75(\mathrm{~s}, 1 \mathrm{H}), 7.58$ (t, $J=7.2 \mathrm{~Hz}$, 
1H), 7.50 (d, $J=7.2 \mathrm{~Hz}, 1 \mathrm{H}), 7.48$ (d, $J=7.2 \mathrm{~Hz}, 1 \mathrm{H}), 7.41$ (s, 1H), 7.31 (d, $J=8.8 \mathrm{~Hz}, 1 \mathrm{H}), 7.17$ (d, $J=$ $8.8 \mathrm{~Hz}, 1 \mathrm{H}), 5.57(\mathrm{~d}, J=2.0 \mathrm{~Hz}, 1 \mathrm{H}), 4.24-4.17(\mathrm{~m}, 2 \mathrm{H}), 3.57$ (s, 3H), $3.34(\mathrm{~d}, J=8.8 \mathrm{~Hz}, 1 \mathrm{H}), 2.53$ (s, $2 \mathrm{H}), 2.18(\mathrm{~s}, 3 \mathrm{H}), 1.34$ (s, 3H), $1.08(\mathrm{~s}, 3 \mathrm{H}) ;{ }^{13} \mathrm{C} \mathrm{NMR}\left(100 \mathrm{MHz}, \mathrm{CD}_{2} \mathrm{Cl}_{2}\right) \delta$ 159.1, 156.9, 150.1, 139.2, 134.0, 129.7 (2C), 127.6 (2C), 126.0, 125.7, 120.9, 114.7, 113.5, 111.6, 98.3, 84.5, 78.9, 71.6, 69.0, 62.1, 29.2, 22.7, 8.4; IR (film) $v_{\max } 3439,3429,2982,2930,2853,1713,1630,1609,1499,1464,1448,1369$, $1327,1285,1261,1167,1113,1088 \mathrm{~cm}^{-1}$; HRMS (ESI +$) \mathrm{m} / \mathrm{z} 506.1490\left(\mathrm{M}+\mathrm{H}^{+}, \mathrm{C}_{24} \mathrm{H}_{28} \mathrm{NO}_{9} \mathrm{~S}\right.$ requires $\mathrm{m} / \mathrm{z}$ 506.1485).<smiles>CO[C@H]1[C@@H](O)[C@@H](O)[C@H](Oc2ccc3cc(NC(=O)Cc4ccccc4)c(=O)oc3c2C)OC1(C)C</smiles>

22

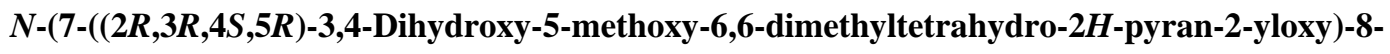
methyl-2-oxo-2H-chromen-3-yl)-2-phenylacetamide (22). Prepared by the general cyclic carbonate solvolysis procedure described above to afford $22(87 \%)$ as a colorless solid: $[\alpha]^{24}=-15.1^{\circ}(\mathrm{c}=0.37$, $\left.\mathrm{CH}_{2} \mathrm{Cl}_{2}\right) ;{ }^{1} \mathrm{H}$ NMR $\left(800 \mathrm{MHz}, \mathrm{CD}_{2} \mathrm{Cl}_{2}\right) \delta 8.58(\mathrm{~s}, 1 \mathrm{H}), 8.04(\mathrm{~s}, 1 \mathrm{H}), 7.40-7.31(\mathrm{~m}, 5 \mathrm{H}), 7.29(\mathrm{~d}, J=8.8$ $\mathrm{Hz}, 1 \mathrm{H}), 7.15(\mathrm{~d}, J=8.8 \mathrm{~Hz}, 1 \mathrm{H}), 5.56(\mathrm{~d}, J=0.8 \mathrm{~Hz}, 1 \mathrm{H}), 4.24-4.20(\mathrm{~m}, 2 \mathrm{H}), 3.76(\mathrm{~s}, 2 \mathrm{H}), 3.57(\mathrm{~s}, 3 \mathrm{H})$, $3.34(\mathrm{~d}, J=8.8 \mathrm{~Hz}, 1 \mathrm{H}), 2.95(\mathrm{~s}, 1 \mathrm{H}), 2.83(\mathrm{~s}, 1 \mathrm{H}), 2.22(\mathrm{~s}, 3 \mathrm{H}), 1.31$ (s, 3H), $1.10(\mathrm{~s}, 3 \mathrm{H}) ;{ }^{13} \mathrm{C}$ NMR $(200$ $\left.\mathrm{MHz}, \mathrm{CD}_{2} \mathrm{Cl}_{2}\right) \delta 171.9,160.7,157.9,151.0,136.2,131.3$ (2C), 130.9 (2C), 129.4, 127.5, 125.9, 123.5, 116.0, 115.7, 113.0, 99.8, 86.1, 80.4, 73.1, 70.5, 63.6, 46.5, 30.8, 24.2, 10.0; IR (film) $v_{\max } 3367,3339$, 3086, 3063, 3030, 2980, 2932, 2853, 2831, 1715, 1684, 1607, 1526, 1369, 1263, 1113, $1084 \mathrm{~cm}^{-1}$; HRMS (ESI+) $m / z$ 484.1982 $\left(\mathrm{M}+\mathrm{H}^{+}, \mathrm{C}_{26} \mathrm{H}_{30} \mathrm{NO}_{8}\right.$ requires $m / z$ 484.1971).<smiles>CO[C@H]1[C@@H](Oc2ccc3cc(NC(=O)Cc4ccccc4)c(=O)oc3c2C)OC(C)(C)[C@H]1OC(=O)O</smiles>

22a

$N$-(7-((3aR,4R,7R,7aR)-7-Methoxy-6,6-dimethyl-2-oxotetrahydro-3aH-[1,3]dioxolo[4,5-

clpyran-4-yloxy)-8-methyl-2-oxo-2H-chromen-3-yl)-2-phenylacetamide (22a). $\quad N$-(3-Dimethylaminopropyl)- $N$ '-ethylcarbodiimide hydrochloride ( $34 \mathrm{mg}, 72 \mu \mathrm{mol}$ ) was added to a solution of aminocoumarin 7 (28 mg, $72 \mu \mathrm{mol}$ ) and phenyl acetic acid ( $24 \mathrm{mg}, 179 \mu \mathrm{mol}$ ) in $\mathrm{CH}_{2} \mathrm{Cl}_{2}$ at rt. The solution was stirred for $14 \mathrm{~h}$, concentrated and the residue purified via preparative $\mathrm{TLC}\left(\mathrm{SiO}_{2}, 40: 1 ; \mathrm{CH}_{2} \mathrm{Cl}_{2}\right.$ :acetone) to afford the $24 \mathrm{mg}(66 \%)$ of 22a as a colorless solid: $[\alpha]^{24}{ }_{\mathrm{D}}=-28.5^{\circ}\left(\mathrm{c}=0.39, \mathrm{CH}_{2} \mathrm{Cl}_{2}\right) ;{ }^{1} \mathrm{H} \mathrm{NMR}\left(800 \mathrm{MHz}, \mathrm{CD}_{2} \mathrm{Cl}_{2}\right)$ $\delta 8.62(\mathrm{~s}, 1 \mathrm{H}), 8.00(\mathrm{~s}, 1 \mathrm{H}), 7.36(\mathrm{t}, J=8.0 \mathrm{~Hz}, 2 \mathrm{H}), 7.33-7.26(\mathrm{~m}, 4 \mathrm{H}), 7.08(\mathrm{~d}, J=8.8 \mathrm{~Hz}, 1 \mathrm{H}), 5.74(\mathrm{~d}$, $J=1.6 \mathrm{~Hz}, 1 \mathrm{H}), 5.01(\mathrm{dd}, J=1.6,8.0 \mathrm{~Hz}, 1 \mathrm{H}), 4.92(\mathrm{t}, J=8.0 \mathrm{~Hz}, 1 \mathrm{H}), 3.72(\mathrm{~s}, 2 \mathrm{H}), 3.53(\mathrm{~s}, 3 \mathrm{H}), 3.30$ (d, $J=8.0 \mathrm{~Hz}, 1 \mathrm{H}), 2.22(\mathrm{~s}, 3 \mathrm{H}), 1.31(\mathrm{~s}, 3 \mathrm{H}), 1.15(\mathrm{~s}, 3 \mathrm{H}) ;{ }^{13} \mathrm{C} \mathrm{NMR}\left(200 \mathrm{MHz}, \mathrm{CD}_{2} \mathrm{Cl}_{2}\right) \delta 171.8,160.6$, 
157.0, 155.1, 151.0, 136.2, 131.3 (2C), 130.9 (2C), 129.4, 127.6, 125.4, 124.0, 116.6, 116.5, 113.1, 96.3, 84.7, 79.9, 79.0, 78.5, 62.3, 46.5, 29.2, 23.9, 10.0; IR (film) $v_{\max } 3333,3088,3063,3030,2984,2935$, 2851, 1809, 1717, 1684, 1609, 1522, 1369, 1261, 1173, 1111, 1088, $1036 \mathrm{~cm}^{-1}$; HRMS (ESI+) $\mathrm{m} / \mathrm{z}$ $510.1755\left(\mathrm{M}+\mathrm{H}^{+}, \mathrm{C}_{27} \mathrm{H}_{28} \mathrm{NO}_{9}\right.$ requires $m / z$ 510.1764).<smiles>CO[C@H]1[C@@H](O)[C@@H](O)C(Oc2ccc3cc(NC(=O)CCc4ccccc4)c(=O)oc3c2C)OC1(C)C</smiles>

23

$N$-(7-((2R,3R,4S,5R)-3,4-Dihydroxy-5-methoxy-6,6-dimethyltetrahydro-2H-pyran-2-yloxy)-8methyl-2-oxo-2H-chromen-3-yl)-3-phenylpropanamide (23). Prepared by the general cyclic carbonate solvolysis procedure described above to afford $23(16 \mathrm{mg}, 79 \%)$ as a colorless solid: $[\alpha]^{24}=-14.6^{\circ}(\mathrm{c}=$ 0.49, $\mathrm{CH}_{2} \mathrm{Cl}_{2}$ ); ${ }^{1} \mathrm{H}$ NMR (800 MHz, $\left.\mathrm{CD}_{2} \mathrm{Cl}_{2}\right) \delta 8.60(\mathrm{~s}, 1 \mathrm{H}), 7.95(\mathrm{~s}, 1 \mathrm{H}), 7.31-7.24(\mathrm{~m}, 3 \mathrm{H}), 7.23(\mathrm{~d}, J=$ $7.2 \mathrm{~Hz}, 2 \mathrm{H}), 7.18(\mathrm{t}, J=8.7 \mathrm{~Hz}, 1 \mathrm{H}), 7.15(\mathrm{~d}, J=8.8 \mathrm{~Hz}, 1 \mathrm{H}), 5.56(\mathrm{~d}, J=2.4 \mathrm{~Hz}, 1 \mathrm{H}), 4.23-4.17(\mathrm{~m}$, 2H), 3.56 (s, 3H), 3.33 (d, $J=8.8 \mathrm{~Hz}, 1 \mathrm{H}), 3.01$ (t, $J=8.0 \mathrm{~Hz}, 2 \mathrm{H}), 2.85(\mathrm{~s}, 1 \mathrm{H}), 2.76(\mathrm{~s}, 1 \mathrm{H}), 2.72(\mathrm{t}, J=$ $8.0 \mathrm{~Hz}, 2 \mathrm{H}), 2.22(\mathrm{~s}, 3 \mathrm{H}), 1.33(\mathrm{~s}, 3 \mathrm{H}), 1.10(\mathrm{~s}, 3 \mathrm{H}) ;{ }^{13} \mathrm{C} \mathrm{NMR}\left(200 \mathrm{MHz}, \mathrm{CD}_{2} \mathrm{Cl}_{2}\right) \delta 173.2,160.8,157.8$, 150.9, 142.5, 130.4 (2C), 130.2 (2C), 128.2, 127.5, 125.8, 123.6, 116.0, 115.8, 113.0, 99.8, 86.1, 80.4, 73.1, 70.5, 63.6, 40.9, 33.0, 30.8, 24.2, 10.0; IR (film) $v_{\max } 3427,3391,3325,3080,3086,3061,2932,2833$, $1709,1684,1607,1529,1377,1223,1113,1084 \mathrm{~cm}^{-1}$; HRMS (ESI+) $\mathrm{m} / \mathrm{z} 498.2140\left(\mathrm{M}+\mathrm{H}^{+}, \mathrm{C}_{27} \mathrm{H}_{32} \mathrm{NO}_{8}\right.$ requires $\mathrm{m} / \mathrm{z} 498.2128)$.<smiles>COC1[C@H]2OC(=O)O[C@H]2[C@H](Oc2ccc3cc(NC(=O)CCc4ccccc4)c(=O)oc3c2C)OC1(C)C</smiles>

23a

$N$-(7-((3aR,4R,7R,7aR)-7-Methoxy-6,6-dimethyl-2-oxotetrahydro-3aH-[1,3]dioxolo[4,5-

c]pyran-4-yloxy)-8-methyl-2-oxo-2H-chromen-3-yl)-3-phenylpropanamide (23a). Prepared by the general EDCI coupling procedure described above to afford 23a $(21 \mathrm{mg}, 58 \%)$ as a colorless solid: $[\alpha]^{23}$ $=-23.4^{\circ}\left(\mathrm{c}=0.32,20 \% \mathrm{MeOH}\right.$ in $\left.\mathrm{CH}_{2} \mathrm{Cl}_{2}\right) ;{ }^{1} \mathrm{H}$ NMR $\left(400 \mathrm{MHz}, \mathrm{CD}_{2} \mathrm{Cl}_{2}\right) \delta 8.65(\mathrm{~s}, 1 \mathrm{H}), 7.97(\mathrm{~s}, 1 \mathrm{H}), 7.36$ (d, $J=8.7 \mathrm{~Hz}, 1 \mathrm{H}), 7.32-7.13(\mathrm{~m}, 5 \mathrm{H}), 7.15$ (d, $J=8.7 \mathrm{~Hz}, 1 \mathrm{H}), 5.81$ (d, $J=2.1 \mathrm{~Hz}, 1 \mathrm{H}), 5.08$ (dd, $J=$ 2.1, $8.0 \mathrm{~Hz}, 1 \mathrm{H}), 4.98$ (t, $J=8.0 \mathrm{~Hz}, 1 \mathrm{H}), 3.60(\mathrm{~s}, 3 \mathrm{H}), 3.35$ (d, $J=8.0 \mathrm{~Hz}, 1 \mathrm{H}), 3.04$ (t, $J=7.7 \mathrm{~Hz}, 2 \mathrm{H})$, $2.75(\mathrm{t}, J=7.7 \mathrm{~Hz}, 2 \mathrm{H}), 2.28(\mathrm{~s}, 3 \mathrm{H}), 1.36(\mathrm{~s}, 3 \mathrm{H}), 1.20(\mathrm{~s}, 3 \mathrm{H}) ;{ }^{13} \mathrm{C} \mathrm{NMR}\left(125 \mathrm{MHz}, \mathrm{CD}_{2} \mathrm{Cl}_{2}\right) \delta 170.4$, 158.0, 154.3, 152.4, 148.3, 139.9, 128.1 (2C), 127.7 (2C), 125.4, 124.8, 122.7, 121.4, 113.9 (2C), 110.4, 93.6, 82.0, 77.2, 76.4, 75.8, 59.6, 38.7, 31.2, 28.6, 21.9, 7.3; IR (film) $v_{\max } 3327,3086,3063,3026,2982$, 
2930, 2851, 1811, 1717, 1684, 1607, 1522, 1371, 1259, 1173, 1111, 1086, 1036, $1005 \mathrm{~cm}^{-1}$; HRMS (ESI+) $\mathrm{m} / \mathrm{z} 524.1912\left(\mathrm{M}+\mathrm{H}^{+}, \mathrm{C}_{28} \mathrm{H}_{30} \mathrm{NO}_{9}\right.$ requires $\left.\mathrm{m} / \mathrm{z} 524.1921\right)$.<smiles>CO[C@H]1[C@@H](O)[C@@H](O)C(Oc2ccc3cc(NC(=O)OCc4ccccc4)c(=O)oc3c2C)OC1(C)C</smiles>

24

Benzyl 7-((2R,3R,4S,5R)-3,4-dihydroxy-5-methoxy-6,6-dimethyltetrahydro-2H-pyran-2yloxy)-8-methyl-2-oxo-2H-chromen-3-ylcarbamate (24). $\mathrm{Et}_{3} \mathrm{~N}$ (10\% total volume) was added dropwise to a solution of cyclic carbonate $7 \mathbf{a}(25 \mathrm{mg}, 48 \mu \mathrm{mol})$ in methanol $(0.6 \mathrm{~mL})$ at $\mathrm{rt}$. The resulting mixture was stirred for $14 \mathrm{~h}$ and then concentrated. The residue was purified via preparative TLC $\left(\mathrm{SiO}_{2}, 4: 1\right.$; $\mathrm{CH}_{2} \mathrm{Cl}_{2}$ :acetone) to afford $24(19 \mathrm{mg}, 82 \%)$ as a white solid: $[\alpha]^{25}=-11.3^{\circ}(\mathrm{c}=0.84,20 \% \mathrm{MeOH}$ in $\mathrm{CH}_{2} \mathrm{Cl}_{2}$ ); ${ }^{1} \mathrm{H}$ NMR (500 MHz, 20\% $\mathrm{CD}_{3} \mathrm{OD}$ in $\mathrm{CD}_{2} \mathrm{Cl}_{2}$ ) $\delta 8.17(\mathrm{~s}, 1 \mathrm{H}), 7.35-7.21(\mathrm{~m}, 6 \mathrm{H}), 7.10(\mathrm{~d}, J=9.0$ $\mathrm{Hz}, 1 \mathrm{H}), 5.46(\mathrm{~d}, J=2.5 \mathrm{~Hz}, 1 \mathrm{H}), 5.13(\mathrm{~s}, 2 \mathrm{H}), 4.07-4.00(\mathrm{~m}, 2 \mathrm{H}), 3.56(\mathrm{~s}, 3 \mathrm{H}), 3.25(\mathrm{~d}, J=9.0 \mathrm{~Hz}, 1 \mathrm{H})$, 2.17 (s, 3H), 1.24 (s, 3H), 1.01 (s, 3H); ${ }^{13} \mathrm{C}$ NMR (125 MHz, 20\% $\mathrm{CD}_{3} \mathrm{OD}$ in $\left.\mathrm{CD}_{2} \mathrm{Cl}_{2}\right) \delta$ 157.1, 154.1, 151.7, 147.1, 134.2, 126.7 (2C), 126.5, 126.3 (2C), 123.5, 120.9, 120.0, 112.2, 112.0, 109.3, 96.7, 82.3, 76.7, 69.5, 66.6, 65.5, 59.7, 26.7, 20.4, 6.0; IR (film) $v_{\max } 3443,3421,2982,2936,2836,2525,1701,1632$, $1609,1456,1416,1360,1288,1115,1086 \mathrm{~cm}^{-1}$; HRMS (ESI+) $\mathrm{m} / z$ 522.1721 $\left(\mathrm{M}+\mathrm{Na}^{+}, \mathrm{C}_{26} \mathrm{H}_{29} \mathrm{NO}_{9} \mathrm{Na}\right.$ requires $\mathrm{m} / \mathrm{z} 522.1740)$.<smiles>CO[C@H]1[C@@H](O)[C@@H](O)C(Oc2ccc3cc(NC(=O)/C=C/c4ccccc4)c(=O)oc3c2C)OC1(C)C</smiles>

25

$N$-(7-((2R,3R,4S,5R)-3,4-Dihydroxy-5-methoxy-6,6-dimethyltetrahydro-2H-pyran-2-yloxy)-8methyl-2-oxo-2H-chromen-3-yl)cinnamamide (25). $\quad N$-(3-Dimethylamino-propyl)- $N$ '-ethylcarbodiimide hydrochloride (44 mg, $227 \mu \mathrm{mol})$ was added to a solution of aminocoumarin 7 (36 mg, $91 \mu \mathrm{mol})$ and transcinnamic acid (27 mg, $182 \mu \mathrm{mol}$ ) in $\mathrm{CH}_{2} \mathrm{Cl}_{2}$ containing 30\% pyridine at rt. The solution was stirred for 14 h, concentrated and the residue purified via preparative TLC $\left(\mathrm{SiO}_{2}, 40: 1 ; \mathrm{CH}_{2} \mathrm{Cl}_{2}\right.$ :acetone) to afford the 30 $\mathrm{mg}(63 \%)$ of the amide as an off-white solid. The cyclic carbonate $(30 \mathrm{mg})$ was dissolved in $0.8 \mathrm{~mL}$ of methanol and $0.1 \mathrm{~mL}$ of $\mathrm{Et}_{3} \mathrm{~N}$ was added dropwise. The reaction mixture was stirred for $14 \mathrm{~h}$ and then was concentrated. The residue was purified by preparative TLC (10:1; $\mathrm{CH}_{2} \mathrm{Cl}_{2}$ :methanol) to afford 25 (29 $\mathrm{mg}$, $86 \%)$ as a colorless solid: $[\alpha]^{26}=-41.7^{\circ}(\mathrm{c}=0.18$, DMSO $) ;{ }^{1} \mathrm{H}$ NMR $\left(500 \mathrm{MHz}, 20 \% \mathrm{CD}_{3} \mathrm{OD}\right.$ in 
$\left.\mathrm{CD}_{2} \mathrm{Cl}_{2}\right) \delta 8.69(\mathrm{~s}, 1 \mathrm{H}), 7.66(\mathrm{~d}, J=16.0 \mathrm{~Hz}, 1 \mathrm{H}), 7.56(\mathrm{~d}, J=6.5 \mathrm{~Hz}, 2 \mathrm{H}), 7.38-7.30(\mathrm{~m}, 4 \mathrm{H}), 7.17(\mathrm{~d}, J$ $=8.5 \mathrm{~Hz}, 1 \mathrm{H}), 6.80(\mathrm{~d}, J=16.0 \mathrm{~Hz}, 1 \mathrm{H}), 5.51(\mathrm{~s}, 1 \mathrm{H}), 4.19-4.02(\mathrm{~m}, 2 \mathrm{H}), 3.53(\mathrm{~s}, 3 \mathrm{H}), 3.30(\mathrm{~d}, J=9.0$ $\mathrm{Hz}, 1 \mathrm{H}), 2.23$ (s, 3H), 1.28 (s, 3H), $1.06(\mathrm{~s}, 3 \mathrm{H}) ;{ }^{13} \mathrm{C} \mathrm{NMR}\left(125 \mathrm{MHz}, 20 \% \mathrm{CD}_{3} \mathrm{OD}\right.$ in $\left.\mathrm{CD}_{2} \mathrm{Cl}_{2}\right) \delta 164.8$, 157.9, 155.7, 148.8, 140.8, 134.7, 131.9, 129.0 (2C), 127.8 (2C), 125.9, 124.0, 122.1, 122.0, 113.3, 112.8, 110.8, 98.5, 83.4, 77.8, 70.8, 67.8, 61.0, 28.5, 22.9, 8.1; IR (film) $v_{\max } 3447,3412,3385,3071,3059,2924$, 2853, 1701, 1609, 1412, 1373, 1262, 1180, 1113, 1082, 1059, $1022 \mathrm{~cm}^{-1}$; HRMS (ESI+) m/z 496.1967 (M $+\mathrm{H}^{+}, \mathrm{C}_{27} \mathrm{H}_{30} \mathrm{NO}_{8}$ requires $\mathrm{m} / \mathrm{z}$ 496.1971).<smiles>COc1ccc(C(=O)O)cc1I</smiles>

\section{6}

3-Iodo-4-methoxybenzoic acid (26). Lithium hydroxide (72 $\mathrm{mg}, 1.71 \mathrm{mmol})$ was added to a mixture of methyl 3-iodo-4-methoxybenzoate $(100 \mathrm{mg}, 0.342 \mathrm{mmol})$ in $3.0 \mathrm{~mL}$ of a 3:1:1 THF-MeOHwater solution at rt. The mixture was stirred for $8 \mathrm{~h}$ in the dark and than diluted with $\mathrm{H}_{2} \mathrm{O}(2 \mathrm{~mL})$. The solution was acidified to $\mathrm{pH}=2$ by the dropwise addition of concentrated $\mathrm{HCl}$. The solution was extracted twice with EtOAc (10 mL portions) and the combined organic layers dried $\left(\mathrm{Na}_{2} \mathrm{SO}_{4}\right)$, filtered, and concentrated to afford acid 26 (95 mg, 100\%) as a yellow solid that was suitable for use without further purification: ${ }^{1} \mathrm{H}$ NMR (400 MHz, DMSO) $\delta 8.24$ (d, $\left.J=2.0 \mathrm{~Hz}, 1 \mathrm{H}\right), 7.92(\mathrm{dd}, J=2.0,8.7 \mathrm{~Hz}, 1 \mathrm{H}), 7.07$ (d, $J=8.7 \mathrm{~Hz}, 1 \mathrm{H}), 3.88(\mathrm{~s}, 3 \mathrm{H})$.

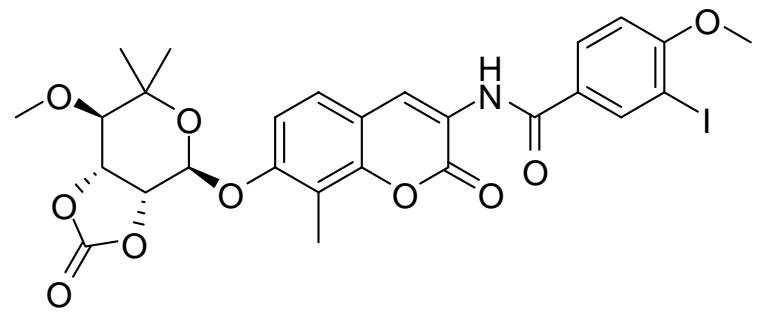

27

\section{3-Iodo-4-methoxy- $N$-(7-((3aR,4R,7R,7aR)-7-methoxy-6,6-dimethyl-2-oxotetrahydro-3aH-}

[1,3]dioxolo[4,5-c]pyran-4-yloxy)-8-methyl-2-oxo-2H-chromen-3-yl)benzamide (27). Prepared by the procedure used for compound 24 to afford $27(96 \%)$ as a yellow solid: $[\alpha]^{25}=-13.9^{\circ}\left(\mathrm{c}=0.17, \mathrm{CH}_{2} \mathrm{Cl}_{2}\right)$; ${ }^{1} \mathrm{H}$ NMR (400 MHz, DMSO) $\delta 9.69$ (s, 1H), 8.47 (d, $\left.J=2.0 \mathrm{~Hz}, 1 \mathrm{H}\right), 8.37$ (d, $\left.J=2.0 \mathrm{~Hz}, 1 \mathrm{H}\right), 8.00$ (dd, $J=$ 2.0, $8.6 \mathrm{~Hz}, 1 \mathrm{H}), 7.60(\mathrm{~d}, J=8.6 \mathrm{~Hz}, 1 \mathrm{H}), 7.17(\mathrm{~d}, J=8.8 \mathrm{~Hz}, 1 \mathrm{H}), 7.12(\mathrm{~d}, J=8.8 \mathrm{~Hz}, 1 \mathrm{H}), 6.08(\mathrm{~d}, J=$ $3.3 \mathrm{~Hz}, 1 \mathrm{H}), 5.20-5.11(\mathrm{~m}, 2 \mathrm{H}), 3.91(\mathrm{~s}, 3 \mathrm{H}), 3.83-3.75(\mathrm{~m}, 1 \mathrm{H}), 3.49(\mathrm{~s}, 3 \mathrm{H}), 2.23(\mathrm{~s}, 3 \mathrm{H}), 1.29(\mathrm{~s}, 3 \mathrm{H})$, $1.12(\mathrm{~s}, 3 \mathrm{H}) ;{ }^{13} \mathrm{C}$ NMR $(100 \mathrm{MHz}$, DMSO) $\delta 164.9,161.5,158.8,156.0,154.4,150.3,139.3,130.8,129.6$, $128.2,126.9,122.7,114.8,114.6,112.1,111.8,94.2,86.7,82.3,78.5,77.2,76.9,60.7,57.6,27.7,23.6$, 
9.0; IR (film) $v_{\max } 3406,3096,3067,2982,2937,2843,1811,1701,1670,1607,1593,1526,1487,1367$, 1256, 1171, 1095, 1078, 1038, $1007 \mathrm{~cm}^{-1}$; HRMS (ESI+) $\mathrm{m} / \mathrm{z} 652.0691\left(\mathrm{M}+\mathrm{H}^{+}, \mathrm{C}_{27} \mathrm{H}_{27} \mathrm{NO}_{10} \mathrm{I}\right.$ requires $\mathrm{m} / \mathrm{z}$ $652.0680)$.

General procedure for Suzuki coupling and solvolysis of the cyclic carbonate: Aryl iodide 27 (1.0 eq), $2 \mathrm{M} \mathrm{K}_{2} \mathrm{CO}_{3(\mathrm{aq})}(3.0 \mathrm{eq})$ and the aryl boronic acid were dissolved in dioxane before $\mathrm{PdCl}_{2}(\mathrm{dppf}) \cdot \mathrm{CHCl}_{3}(3$ mol\%) was added to the solution at $\mathrm{rt}$. The resulting solution was stirred at $\mathrm{rt}$ for $30 \mathrm{~min}$ and then warmed to $55^{\circ} \mathrm{C}$ for $3-16 \mathrm{~h}$. After which, the mixture was concentrated, filtered through a pad of silica gel (eluted with 40:1; $\mathrm{CH}_{2} \mathrm{Cl}_{2}$ :acetone) and purified via preparative TLC $\left(\mathrm{SiO}_{2}, 40: 1 ; \mathrm{CH}_{2} \mathrm{Cl}_{2}\right.$ :acetone). The resulting product was dissolved in methanol containing $10 \% \mathrm{Et}_{3} \mathrm{~N}$ and stirred for $14 \mathrm{~h}$ before concentrating. The residue was purified by preparative TLC $\left(4: 1 ; \mathrm{CH}_{2} \mathrm{Cl}_{2}\right.$ :acetone) to afford the corresponding diol.<smiles>COc1ccc(C(=O)Nc2cc3ccc(O[C@@H]4OC(C)(C)[C@@H](OC)[C@H](O)[C@H]4O)c(C)c3oc2=O)cc1-c1ccccc1</smiles>

28

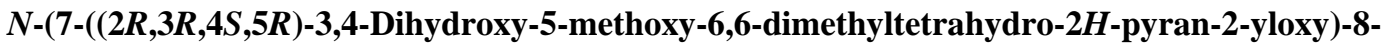
methyl-2-oxo-2H-chromen-3-yl)-6-methoxybiphenyl-3-carboxamide (28). Colorless solid (46\%, 2 steps): $[\alpha]_{\mathrm{D}}^{25}=-8.7^{\circ}\left(\mathrm{c}=0.23, \mathrm{CH}_{2} \mathrm{Cl}_{2}\right) ;{ }^{1} \mathrm{H}$ NMR $\left(800 \mathrm{MHz}, \mathrm{CD}_{2} \mathrm{Cl}_{2}\right) \delta 8.78(\mathrm{~s}, 1 \mathrm{H}), 8.69(\mathrm{~s}, 1 \mathrm{H}), 7.93$ $(\mathrm{dd}, J=4.4,8.0 \mathrm{~Hz}, 1 \mathrm{H}), 7.87(\mathrm{~d}, J=2.4 \mathrm{~Hz}, 1 \mathrm{H}), 7.55(\mathrm{~d}, J=8.0 \mathrm{~Hz}, 2 \mathrm{H}), 7.45(\mathrm{t}, J=8.0 \mathrm{~Hz}, 2 \mathrm{H}), 7.93$ $(\mathrm{dd}, J=4.4,8.0 \mathrm{~Hz}, 2 \mathrm{H}), 7.21(\mathrm{~d}, J=8.8 \mathrm{~Hz}, 1 \mathrm{H}), 7.10(\mathrm{~d}, J=8.8 \mathrm{~Hz}, 2 \mathrm{H}), 5.60(\mathrm{~s}, 1 \mathrm{H}), 4.27-4.20(\mathrm{~m}$, 2H), $3.90(\mathrm{~s}, 3 \mathrm{H}), 3.59$ (s, 3H), 3.36 (d, J = 9.6 Hz, 1H), 2.73 (s, 2H), 2.28 (s, 3H), $1.36(\mathrm{~s}, 3 \mathrm{H}), 1.13$ (s, $3 \mathrm{H}) ;{ }^{13} \mathrm{C} \mathrm{NMR}\left(200 \mathrm{MHz}, \mathrm{CD}_{2} \mathrm{Cl}_{2}\right) \delta 167.2,161.7,161.2,157.9,151.0,139.4,133.0,131.8,131.5$ (3C), 130.0 (2C), 129.4, 128.1, 127.6, 125.8, 124.0, 116.1, 116.0, 113.0 (2C), 99.7, 86.1, 80.4, 73.1, 70.5, 63.7, 57.8, 30.8, 24.2, 10.1; IR (film) $v_{\max }$ 3402, 3086, 3055, 3028, 2974, 2934, 2849, 2837, 1709, 1670, 1607, 1526, 1504, 1489, 1367, 1265, 1231, $1095 \mathrm{~cm}^{-1}$; HRMS (ESI+) m/z 576.2231 (M + $\mathrm{H}^{+}, \mathrm{C}_{32} \mathrm{H}_{34} \mathrm{NO}_{9}$ requires $m / z$ 576.2234).<smiles>COc1ccc(C(=O)Nc2cc3ccc(O[C@@H]4OC(C)(C)[C@@H](OC)[C@H](O)[C@H]4O)c(C)c3oc2=O)cc1-c1ccccc1C</smiles> 
$N$-(7-((2R,3R,4S,5R)-3,4-Dihydroxy-5-methoxy-6,6-dimethyltetrahydro-2H-pyran-2-yloxy)-8methyl-2-oxo-2H-chromen-3-yl)-6-methoxy-2'-methylbiphenyl-3-carboxamide (29). Colorless solid (45\%, 2 steps): $[\alpha]^{24}{ }_{\mathrm{D}}=-17.0^{\circ}\left(\mathrm{c}=0.23, \mathrm{CH}_{2} \mathrm{Cl}_{2}\right) ;{ }^{1} \mathrm{H}$ NMR $\left(400 \mathrm{MHz}, \mathrm{CD}_{2} \mathrm{Cl}_{2}\right) \delta 8.78(\mathrm{~s}, 1 \mathrm{H}), 8.68(\mathrm{~s}$, 1H), 7.98 (dd, $J=2.4,8.6 \mathrm{~Hz}, 1 \mathrm{H}), 7.72(\mathrm{~d}, J=2.4 \mathrm{~Hz}, 1 \mathrm{H}), 7.39$ (d, $J=8.7 \mathrm{~Hz}, 1 \mathrm{H}), 7.33-7.16(\mathrm{~m}, 5 \mathrm{H})$, $7.10(\mathrm{~d}, J=8.7 \mathrm{~Hz}, 1 \mathrm{H}), 5.61$ (d, $J=1.8 \mathrm{~Hz}, 1 \mathrm{H}), 4.28-4.22$ (m, 2H), 3.96 (s, 3H), 3.59 (s, 3H), 3.36 (d, $J$ $=8.7 \mathrm{~Hz}, 1 \mathrm{H}), 2.85(\mathrm{~s}, 1 \mathrm{H}), 2.78(\mathrm{~s}, 1 \mathrm{H}), 2.28(\mathrm{~s}, 3 \mathrm{H}), 2.14(\mathrm{~s}, 3 \mathrm{H}), 1.36(\mathrm{~s}, 3 \mathrm{H}), 1.13(\mathrm{~s}, 3 \mathrm{H}) ;{ }^{13} \mathrm{C} \mathrm{NMR}$ $\left(125 \mathrm{MHz}, \mathrm{CD}_{2} \mathrm{Cl}_{2}\right) \delta 165.6,160.4,159.6,156.3,149.4,138.0,137.3,131.6,130.3$ (2C), 130.0, 128.8, 128.1, 126.3, 126.0 (2C), 124.1, 122.4, 114.5, 114.4, 111.5, 111.0, 98.2, 84.6, 78.8, 71.6, 69.0, 62.1, 56.1, 29.3, 22.7, 20.0, 8.5; IR (film) $v_{\max } 3404,3057,2974,2930,2837,1713,1672,1607,1526,1501,1487$, 1367, 1265, 1231, $1094 \mathrm{~cm}^{-1}$; HRMS (ESI+) m/z $590.2390\left(\mathrm{M}+\mathrm{H}^{+}, \mathrm{C}_{33} \mathrm{H}_{36} \mathrm{NO}_{9}\right.$ requires $\left.\mathrm{m} / \mathrm{z} 590.2390\right)$.<smiles>COc1ccc(C(=O)Nc2cc3ccc(O[C@@H]4OC(C)(C)[C@@H](OC)[C@H](O)[C@H]4O)c(C)c3oc2=O)cc1-c1cccc(C)c1</smiles>

30

$N$-(7-((2R,3R,4S,5R)-3,4-Dihydroxy-5-methoxy-6,6-dimethyltetrahydro-2H-pyran-2-yloxy)-8methyl-2-oxo-2H-chromen-3-yl)-6-methoxy-3'-methylbiphenyl-3-carboxamide (30). Colorless solid (46\%, 2 steps): $[\alpha]^{24}=-14.1^{\circ}\left(\mathrm{c}=0.17, \mathrm{CH}_{2} \mathrm{Cl}_{2}\right) ;{ }^{1} \mathrm{H}$ NMR $\left(400 \mathrm{MHz}, \mathrm{CD}_{2} \mathrm{Cl}_{2}\right) \delta 8.79(\mathrm{~s}, 1 \mathrm{H}), 8.70(\mathrm{~s}$, 1H), $7.91(\mathrm{dd}, J=2.4,8.6 \mathrm{~Hz}, 1 \mathrm{H}), 7.86(\mathrm{~d}, J=2.4 \mathrm{~Hz}, 1 \mathrm{H}), 7.41(\mathrm{~d}, J=8.6 \mathrm{~Hz}, 1 \mathrm{H}), 7.36-7.30(\mathrm{~m}, 3 \mathrm{H})$, $7.21(\mathrm{~d}, J=6.0 \mathrm{~Hz}, 2 \mathrm{H}), 7.10(\mathrm{~d}, J=8.7 \mathrm{~Hz}, 1 \mathrm{H}), 5.61(\mathrm{~d}, J=1.9 \mathrm{~Hz}, 1 \mathrm{H}), 4.27-4.20(\mathrm{~m}, 2 \mathrm{H}), 3.90$ (s, 3H), 3.59 (s, 3H), 3.37 (d, J = 8.8 Hz, 1H), 2.80 (s, 2H), 2.43 (s, 3H), 2.29 (s, 3H), 1.35 (s, 3H), 1.14 (s, $3 \mathrm{H}) ;{ }^{13} \mathrm{C}$ NMR $\left(125 \mathrm{MHz}, \mathrm{CD}_{2} \mathrm{Cl}_{2}\right) \delta 164.5,159.1,158.5,155.2,148.3,137.1,136.6,130.4,129.4,129.1$, $127.4,127.2$ (2C), 125.9, 125.3, 124.9, 123.0, 121.3, 113.4, 113.3, 110.3, 110.2, 97.1, 83.4, 77.7, 70.5, 67.8, 61.0, 55.0, 28.1, 21.5, 20.4, 7.3; IR (film) $v_{\max } 3402,3084,3051,2974,2930,2839,1713,1668$, 1607, 1526, 1502, 1367, 1267, 1238, $1092 \mathrm{~cm}^{-1}$; HRMS (ESI+) $\mathrm{m} / \mathrm{z} 590.2390\left(\mathrm{M}+\mathrm{H}^{+}, \mathrm{C}_{33} \mathrm{H}_{36} \mathrm{NO}_{9}\right.$ requires $\mathrm{m} / \mathrm{z} 590.2390)$.<smiles>COc1ccc(C(=O)Nc2cc3ccc(O[C@@H]4OC(C)(C)[C@@H](OC)[C@H](O)[C@H]4O)c(C)c3oc2=O)cc1-c1ccc(C)cc1</smiles> 
$N$-(7-((2R,3R,4S,5R)-3,4-Dihydroxy-5-methoxy-6,6-dimethyltetrahydro-2H-pyran-2-yloxy)-8methyl-2-oxo-2H-chromen-3-yl)-6-methoxy-4'-methylbiphenyl-3-carboxamide (31). Colorless solid (66\%, 2 steps): $[\alpha]^{24}=-16.8^{\circ}\left(\mathrm{c}=0.10, \mathrm{CH}_{2} \mathrm{Cl}_{2}\right) ;{ }^{1} \mathrm{H}$ NMR $\left(500 \mathrm{MHz}, \mathrm{CD}_{2} \mathrm{Cl}_{2}\right) \delta 8.76(\mathrm{~s}, 1 \mathrm{H}), 8.67(\mathrm{~s}$, 1H), 7.88 (dd, $J=2.0,8.5 \mathrm{~Hz}, 1 \mathrm{H}), 7.83(\mathrm{~d}, J=2.0 \mathrm{~Hz}, 1 \mathrm{H}), 7.41$ (d, $J=8.0 \mathrm{~Hz}, 2 \mathrm{H}), 7.36$ (d, $J=8.5 \mathrm{~Hz}$, 1H), $7.24(\mathrm{~d}, J=8.0 \mathrm{~Hz}, 2 \mathrm{H}), 7.18$ (d, $J=8.5 \mathrm{~Hz}, 1 \mathrm{H}), 7.07$ (d, $J=8.5 \mathrm{~Hz}, 1 \mathrm{H}), 5.58$ (s, 1H), $4.25-4.18$ (m, 2H), 3.87 (s, 3H), 3.56 (s, 3H), $3.33(\mathrm{~d}, J=9.0 \mathrm{~Hz}, 1 \mathrm{H}), 2.70$ (s, 1H), 2.65 (s, 1H), 2.39 (s, 3H), 2.26 (s, $3 \mathrm{H}), 1.34(\mathrm{~s}, 3 \mathrm{H}), 1.11(\mathrm{~s}, 3 \mathrm{H}) ;{ }^{13} \mathrm{C} \mathrm{NMR}\left(125 \mathrm{MHz}, \mathrm{CD}_{2} \mathrm{Cl}_{2}\right) \delta 165.7,160.2,159.6,156.3,149.5,137.8$, 134.9, 131.4, 130.1, 129.8 (2C), 129.2 (2C), 128.3, 126.5, 126.1, 124.1, 122.5, 114.5, 114.4, 111.5, 111.3, 98.2, 84.6, 78.8, 71.6, 69.0, 62.1, 56.2, 29.3, 22.7, 21.3, 8.5; IR (film) $v_{\max } 3404,3084,2972,2926,2853$, $2841,1713,1668,1605,1520,1367,1265,1232,1094 \mathrm{~cm}^{-1}$; HRMS (ESI+) $\mathrm{m} / \mathrm{z} 590.2388\left(\mathrm{M}+\mathrm{H}^{+}\right.$, $\mathrm{C}_{33} \mathrm{H}_{36} \mathrm{NO}_{9}$ requires $m / z$ 590.2390).<smiles>COc1ccccc1-c1cc(C(=O)Nc2cc3ccc(O[C@@H]4OC(C)(C)[C@@H](OC)[C@H](O)[C@H]4O)c(C)c3oc2=O)ccc1OC</smiles>

32

$N$-(7-((2R,3R,4S,5R)-3,4-Dihydroxy-5-methoxy-6,6-dimethyltetrahydro-2H-pyran-2-yloxy)-8methyl-2-oxo-2H-chromen-3-yl)-2',6-dimethoxybiphenyl-3-carboxamide (32). Colorless solid (62\%, 2 steps): $[\alpha]^{25}{ }_{\mathrm{D}}=-12.2^{\circ}\left(\mathrm{c}=0.30, \mathrm{CH}_{2} \mathrm{Cl}_{2}\right) ;{ }^{1} \mathrm{H}$ NMR $\left(500 \mathrm{MHz}, \mathrm{CD}_{2} \mathrm{Cl}_{2}\right) \delta 8.75(\mathrm{~s}, 1 \mathrm{H}), 8.66(\mathrm{~s}, 1 \mathrm{H}), 7.92$ $(\mathrm{dd}, J=2.5,9.0 \mathrm{~Hz}, 1 \mathrm{H}), 7.76(\mathrm{~d}, J=2.5 \mathrm{~Hz}, 1 \mathrm{H}), 7.39-7.33(\mathrm{~m}, 2 \mathrm{H}), 7.23(\mathrm{dd}, J=2.0,7.5 \mathrm{~Hz}, 1 \mathrm{H}), 7.18$ (d, $J=8.5 \mathrm{~Hz}, 1 \mathrm{H}), 7.07$ (d, $J=9.0 \mathrm{~Hz}, 1 \mathrm{H}), 7.04-6.98$ (m, 2H), 5.58 (d, $J=2.5 \mathrm{~Hz}, 1 \mathrm{H}), 4.27-4.20$ (m, 2H), 3.84 (s, 3H), 3.77 (s, 3H), 3.57 (s, 3H), 3.33 (d, $J=9.0 \mathrm{~Hz}, 1 \mathrm{H}), 2.75$ (d, $J=2.5 \mathrm{~Hz}, 1 \mathrm{H}), 2.67$ (d, $J=$ $2.5 \mathrm{~Hz}, 1 \mathrm{H}), 2.26(\mathrm{~s}, 3 \mathrm{H}), 1.34(\mathrm{~s}, 3 \mathrm{H}), 1.11(\mathrm{~s}, 3 \mathrm{H}) ;{ }^{13} \mathrm{C} \mathrm{NMR}\left(125 \mathrm{MHz}, \mathrm{CD}_{2} \mathrm{Cl}_{2}\right) \delta$ 164.5, 159.7, 158.4, 156.3, 155.1, 148.3, 130.5, 129.6, 128.4, 127.5 (2C), 125.8, 124.9 (2C), 122.8, 121.3, 119.5, 113.3 (2C), 110.3, 110.2, 110.0, 97.0, 83.4, 77.7, 70.5, 67.8, 61.0, 55.1, 54.8, 28.1, 21.5, 7.3; IR (film) $v_{\max } 3404,3080$, $3057,2930,2835,1709,1670,1607,1526,1502,1487,1367,1265,1244,1094 \mathrm{~cm}^{-1}$; HRMS (ESI+) $\mathrm{m} / \mathrm{z}$ $606.2346\left(\mathrm{M}+\mathrm{H}^{+}, \mathrm{C}_{33} \mathrm{H}_{36} \mathrm{NO}_{10}\right.$ requires $\mathrm{m} / \mathrm{z}$ 606.2339).<smiles>COc1cccc(-c2cc(C(=O)Nc3cc4ccc(O[C@@H]5OC(C)(C)[C@@H](OC)[C@H](O)[C@H]5O)c(C)c4oc3=O)ccc2OC)c1</smiles> 


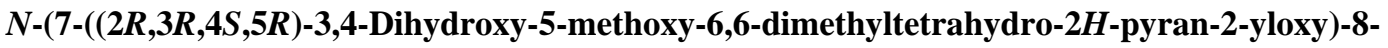
methyl-2-oxo-2H-chromen-3-yl)-3',6-dimethoxybiphenyl-3-carboxamide (33). Colorless solid (52\%, 2 steps): $[\alpha]^{25}=-15.8^{\circ}\left(\mathrm{c}=0.43, \mathrm{CH}_{2} \mathrm{Cl}_{2}\right) ;{ }^{1} \mathrm{H}$ NMR $\left(500 \mathrm{MHz}, \mathrm{CD}_{2} \mathrm{Cl}_{2}\right) \delta 8.76(\mathrm{~s}, 1 \mathrm{H}), 8.68(\mathrm{~s}, 1 \mathrm{H}), 7.91$ $(\mathrm{dd}, J=2.5,8.5 \mathrm{~Hz}, 1 \mathrm{H}), 7.87(\mathrm{~d}, J=2.5 \mathrm{~Hz}, 1 \mathrm{H}), 7.39-7.31(\mathrm{~m}, 2 \mathrm{H}), 7.19(\mathrm{~d}, J=8.5 \mathrm{~Hz}, 1 \mathrm{H}), 7.13-$ $7.05(\mathrm{~m}, 3 \mathrm{H}), 6.92(\mathrm{dd}, J=2.5,8.5 \mathrm{~Hz}, 1 \mathrm{H}), 5.59$ (d, J=2.0 Hz, 1H), $4.28-4.20(\mathrm{~m}, 2 \mathrm{H}), 3.89$ (s, 3H), $3.84(\mathrm{~s}, 3 \mathrm{H}), 3.58(\mathrm{~s}, 3 \mathrm{H}), 3.35(\mathrm{~d}, J=9.0 \mathrm{~Hz}, 1 \mathrm{H}), 2.84(\mathrm{~d}, J=1.5 \mathrm{~Hz}, 1 \mathrm{H}), 2.74(\mathrm{~d}, J=3.0 \mathrm{~Hz}, 1 \mathrm{H}), 2.27$ $(\mathrm{s}, 3 \mathrm{H}), 1.35(\mathrm{~s}, 3 \mathrm{H}), 1.12(\mathrm{~s}, 3 \mathrm{H}) ;{ }^{13} \mathrm{C} \mathrm{NMR}\left(125 \mathrm{MHz}, \mathrm{CD}_{2} \mathrm{Cl}_{2}\right) \delta 164.5,159.0,158.6,158.4,155.2$, $148.3,138.1,130.1,129.1,128.3,127.4,125.3,124.9,123.0,121.3,121.2,114.5,113.3,113.2,112.1$, 110.3, 110.2, 97.1, 83.4, 77.7, 70.4, 67.8, 61.0, 55.1, 54.5, 28.1, 21.5, 7.3; IR (film) $v_{\max } 3404,3078,3057$, 2974, 2934, 2835, 1709, 1670, 1607, 1526, 1502, 1367, 1256, 1244, 1113, 1094, 1051, $1022 \mathrm{~cm}^{-1}$; HRMS (ESI+) $m / z 606.2346\left(\mathrm{M}+\mathrm{H}^{+}, \mathrm{C}_{33} \mathrm{H}_{36} \mathrm{NO}_{10}\right.$ requires $m / z$ 606.2339).

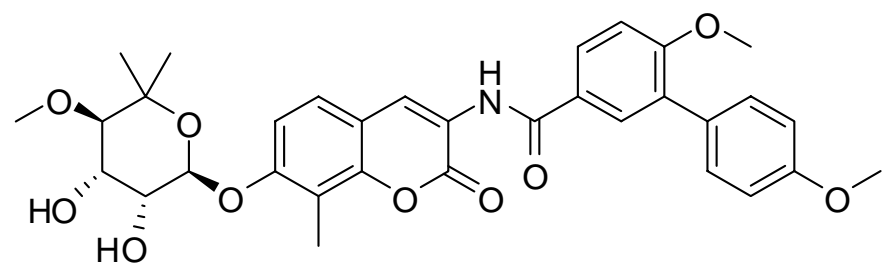

34

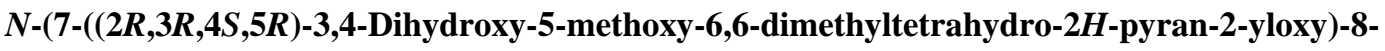
methyl-2-oxo-2H-chromen-3-yl)-4',6-dimethoxybiphenyl-3-carboxamide (34). Colorless solid (46\%, 2 steps): $[\alpha]^{26}{ }_{\mathrm{D}}=-13.9^{\circ}\left(\mathrm{c}=0.49, \mathrm{CH}_{2} \mathrm{Cl}_{2}\right) ;{ }^{1} \mathrm{H} \mathrm{NMR}\left(500 \mathrm{MHz}, \mathrm{CD}_{2} \mathrm{Cl}_{2}\right) \delta 8.74(\mathrm{~s}, 1 \mathrm{H}), 8.66(\mathrm{~s}, 1 \mathrm{H}), 7.86$ $(\mathrm{dd}, J=2.5,8.5 \mathrm{~Hz}, 1 \mathrm{H}), 7.82(\mathrm{~d}, J=2.0 \mathrm{~Hz}, 1 \mathrm{H}), 7.46(\mathrm{dd}, J=2.0,6.5 \mathrm{~Hz}, 2 \mathrm{H}), 7.35(\mathrm{~d}, J=8.5 \mathrm{~Hz}, 1 \mathrm{H})$, $7.17(\mathrm{~d}, J=8.5 \mathrm{~Hz}, 1 \mathrm{H}), 7.05(\mathrm{~d}, J=8.5 \mathrm{~Hz}, 1 \mathrm{H}), 6.96(\mathrm{dd}, J=2.5,6.5 \mathrm{~Hz}, 1 \mathrm{H}), 5.57(\mathrm{~d}, J=2.0 \mathrm{~Hz}, 1 \mathrm{H})$, $4.24-4.17(\mathrm{~m}, 2 \mathrm{H}), 3.86(\mathrm{~s}, 3 \mathrm{H}), 3.83(\mathrm{~s}, 3 \mathrm{H}), 3.56(\mathrm{~s}, 3 \mathrm{H}), 3.33(\mathrm{~d}, J=9.0 \mathrm{~Hz}, 1 \mathrm{H}), 2.25(\mathrm{~s}, 3 \mathrm{H}), 1.34(\mathrm{~s}$, 3H), $1.11(\mathrm{~s}, 3 \mathrm{H}) ;{ }^{13} \mathrm{C}$ NMR $\left(125 \mathrm{MHz}, \mathrm{CD}_{2} \mathrm{Cl}_{2}\right) \delta 164.6,159.0,158.5,158.4,155.1,148.3,129.9$ (3C), 128.9 (2C), 126.9, 125.3, 124.9, 122.9, 121.3, 113.3 (2C), 112.7 (2C), 110.3, 110.1, 97.0, 83.4, 77.7, 70.4, 67.8, 61.0, 55.0, 54.5, 28.1, 21.5, 7.3; IR (film) $v_{\max } 3404,3082,3057,2934,2837,1709,1668,1607$, 1518, 1493, 1265, 1248, 1232, 1118, 1111, 1094, $1080 \mathrm{~cm}^{-1} ;$ HRMS (ESI+) m/z 606.2328 $\left(\mathrm{M}+\mathrm{H}^{+}\right.$, $\mathrm{C}_{33} \mathrm{H}_{36} \mathrm{NO}_{10}$ requires $m / z$ 606.2339).<smiles>COc1ccc(C(=O)Nc2cc3ccc(O[C@@H]4OC(C)(C)[C@@H](OC)[C@H](O)[C@H]4O)c(C)c3oc2=O)cc1-c1ccccc1O</smiles> 


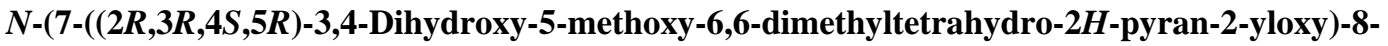
methyl-2-oxo-2H-chromen-3-yl)-2'-hydroxy-6-methoxybiphenyl-3-carboxamide (35). Colorless solid (59\%, 2 steps): $[\alpha]_{\mathrm{D}}^{24}=-13.4^{\circ}\left(\mathrm{c}=0.50,20 \% \mathrm{MeOH}\right.$ in $\left.\mathrm{CH}_{2} \mathrm{Cl}_{2}\right) ;{ }^{1} \mathrm{H} \mathrm{NMR}\left(400 \mathrm{MHz}, \mathrm{CD}_{2} \mathrm{Cl}_{2}\right) \delta 8.73(\mathrm{~s}$, 1H), $8.67(\mathrm{~s}, 1 \mathrm{H}), 7.98(\mathrm{dd}, J=2.3,8.6 \mathrm{~Hz}, 1 \mathrm{H}), 7.88(\mathrm{~d}, J=2.3 \mathrm{~Hz}, 1 \mathrm{H}), 7.39-7.25(\mathrm{~m}, 3 \mathrm{H}), 7.18(\mathrm{~d}, J=$ $8.8 \mathrm{~Hz}, 1 \mathrm{H}), 7.15(\mathrm{~d}, J=8.8 \mathrm{~Hz}, 1 \mathrm{H}), 7.06(\mathrm{dd}, J=1.1,7.4 \mathrm{~Hz}, 1 \mathrm{H}), 7.04(\mathrm{~d}, J=8.1 \mathrm{~Hz}, 1 \mathrm{H}), 5.94$ (s, $1 \mathrm{H})$, $5.57(\mathrm{~d}, J=2.1 \mathrm{~Hz}, 1 \mathrm{H}), 4.26-4.17(\mathrm{~m}, 2 \mathrm{H}), 3.95(\mathrm{~s}, 3 \mathrm{H}), 3.58(\mathrm{~s}, 3 \mathrm{H}), 3.35(\mathrm{~d}, J=9.0 \mathrm{~Hz}, 1 \mathrm{H}), 2.85(\mathrm{~s}$, 1H), $2.75(\mathrm{~s}, 1 \mathrm{H}), 2.27(\mathrm{~s}, 3 \mathrm{H}), 1.36(\mathrm{~s}, 3 \mathrm{H}), 1.10(\mathrm{~s}, 3 \mathrm{H}) ;{ }^{13} \mathrm{C} \mathrm{NMR}\left(125 \mathrm{MHz}, \mathrm{CD}_{2} \mathrm{Cl}_{2}\right) \delta 164.2,158.5$, $158.4,155.2,153.0,148.3,130.6$ (2C), 128.9, 128.0, 126.5, 126.1, 125.0, 124.7, 123.2, 121.0, 120.1, 116.2, 113.3, 113.1, 110.5, 110.3, 96.7, 83.4, 77.7, 70.4, 67.8, 60.9, 55.5, 28.3, 22.2, 8.5; IR (film) $v_{\max } 3400$, 3391, 3090, 2984, 2928, 2849, 1709, 1661, 1651, 1605, 1526, 1495, 1452, 1367, 1267, 1238, 1217, 1180, 1140, $1094 \mathrm{~cm}^{-1}$; HRMS (ESI+) $\mathrm{m} / \mathrm{z} 592.2183\left(\mathrm{M}+\mathrm{H}^{+}, \mathrm{C}_{32} \mathrm{H}_{34} \mathrm{NO}_{10}\right.$ requires $\left.\mathrm{m} / \mathrm{z} 592.2183\right)$.

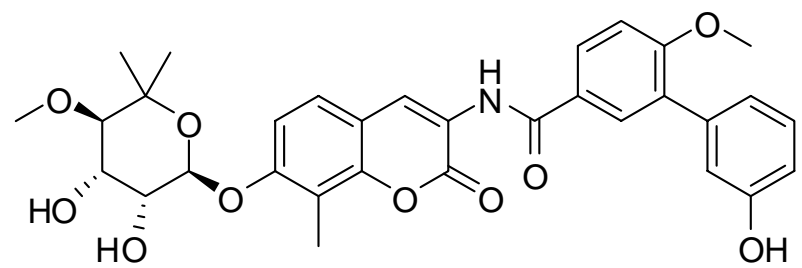

36

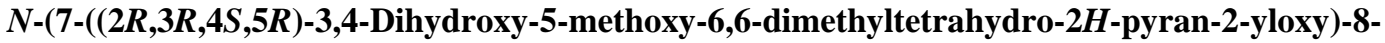
methyl-2-oxo-2H-chromen-3-yl)-3'-hydroxy-6-methoxybiphenyl-3-carboxamide (36). Colorless solid

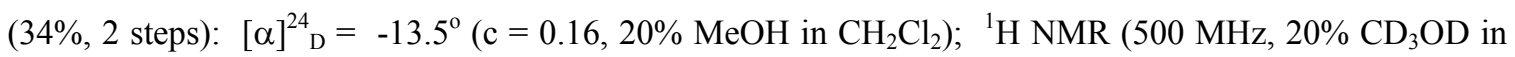
$\left.\mathrm{CD}_{2} \mathrm{Cl}_{2}\right) \delta 8.78(\mathrm{~s}, 1 \mathrm{H}), 8.76(\mathrm{~s}, 1 \mathrm{H}), 7.91(\mathrm{dd}, J=2.4,8.6 \mathrm{~Hz}, 1 \mathrm{H}), 7.86(\mathrm{~d}, J=2.4 \mathrm{~Hz}, 1 \mathrm{H}), 7.40(\mathrm{~d}, J=$ $8.7 \mathrm{~Hz}, 1 \mathrm{H}), 7.29-7.19(\mathrm{~m}, 2 \mathrm{H}), 7.10(\mathrm{~d}, J=8.7 \mathrm{~Hz}, 1 \mathrm{H}), 7.02(\mathrm{dd}, J=1.0,7.6 \mathrm{~Hz}, 1 \mathrm{H}), 6.99(\mathrm{t}, J=2.0$ $\mathrm{Hz}, 1 \mathrm{H}), 6.83(\mathrm{td}, J=1.0,8.0 \mathrm{~Hz}, 1 \mathrm{H}), 5.56(\mathrm{~d}, J=2.1 \mathrm{~Hz}, 1 \mathrm{H}), 4.19-4.09(\mathrm{~m}, 2 \mathrm{H}), 3.89(\mathrm{~s}, 3 \mathrm{H}), 3.58(\mathrm{~s}$, $3 \mathrm{H}), 3.34-3.32(\mathrm{~m}, 1 \mathrm{H}), 2.28(\mathrm{~s}, 3 \mathrm{H}), 1.33(\mathrm{~s}, 3 \mathrm{H}), 1.10(\mathrm{~s}, 3 \mathrm{H}) ;{ }^{13} \mathrm{C} \mathrm{NMR}\left(125 \mathrm{MHz}, 20 \% \mathrm{CD}_{3} \mathrm{OD}\right.$ in $\left.\mathrm{CD}_{2} \mathrm{Cl}_{2}\right) \delta 165.2,159.2,158.8,156.0,155.6,148.5,138.1,130.3,129.1,128.4,127.4,125.2,125.1,124.0$, $121.0,120.2,115.7,113.7,113.4,113.2,110.6,110.4,97.7,83.5,77.8,70.5,67.7,68.9,55.0,27.9,21.6$, 7.2; IR (film) $v_{\max } 3400,2922,2851,1707,1647,1630,1605,1528,1501,1369,1250,1095 \mathrm{~cm}^{-1}$; HRMS (ESI+) $m / z 592.2191\left(\mathrm{M}+\mathrm{H}^{+}, \mathrm{C}_{32} \mathrm{H}_{34} \mathrm{NO}_{10}\right.$ requires $\left.\mathrm{m} / \mathrm{z} 592.2183\right)$. 
<smiles>COc1ccc(C(=O)Nc2cc3ccc(O[C@@H]4OC(C)(C)[C@@H](OC)[C@H](O)[C@H]4O)c(C)c3oc2=O)cc1-c1ccc(O)cc1</smiles>

37

$N$-(7-((2R,3R,4S,5R)-3,4-Dihydroxy-5-methoxy-6,6-dimethyltetrahydro-2H-pyran-2-yloxy)-8methyl-2-oxo-2H-chromen-3-yl)-4'-hydroxy-6-methoxybiphenyl-3-carboxamide (37). Colorless solid (29\%, 2 steps): $[\alpha]^{24}=-21.7^{\circ}\left(\mathrm{c}=0.06,20 \% \mathrm{MeOH}\right.$ in $\left.\mathrm{CH}_{2} \mathrm{Cl}_{2}\right) ;{ }^{1} \mathrm{H} \mathrm{NMR}\left(500 \mathrm{MHz}, 20 \% \mathrm{CD}_{3} \mathrm{OD}\right.$ in $\left.\mathrm{CD}_{2} \mathrm{Cl}_{2}\right) \delta 8.80(\mathrm{~s}, 1 \mathrm{H}), 8.74(\mathrm{~s}, 1 \mathrm{H}), 7.87(\mathrm{dd}, J=2.3,8.7 \mathrm{~Hz}, 1 \mathrm{H}), 7.82(\mathrm{~d}, J=2.3 \mathrm{~Hz}, 1 \mathrm{H}), 7.42-7.35$ (m, 3H), $7.21(\mathrm{~d}, J=8.8 \mathrm{~Hz}, 1 \mathrm{H}), 7.08(\mathrm{~d}, J=8.6 \mathrm{~Hz}, 1 \mathrm{H}), 6.86(\mathrm{~d}, J=8.6 \mathrm{~Hz}, 1 \mathrm{H}), 5.55(\mathrm{~d}, J=2.0 \mathrm{~Hz}$, 1H), 4.16 - 4.09 (m, 2H), 3.88 (s, 3H), 3.57 (s, 3H), 3.33 (d, J = 9.4 Hz, 1H), 2.27 (s, 3H), 1.32 (s, 3H), 1.09 (s, 3H); ${ }^{13} \mathrm{C}$ NMR (125 MHz, 20\% $\mathrm{CD}_{3} \mathrm{OD}$ in $\left.\mathrm{CD}_{2} \mathrm{Cl}_{2}\right) \delta 164.5,158.4,158.0,155.0,154.7,147.6$, 129.4, 129.1 (2C), 128.0, 127.0, 126.0, 124.3, 124.2, 123.2, 120.2, 113.3 (2C), 112.5, 112.3, 109.7, 109.4, 96.9, 82.6, 77.0, 69.7, 66.8, 59.9, 54.1, 27.0, 20.7, 6.3; IR (film) $v_{\max } 3402,3394,2997,2922,2851,1707$, $1653,1605,1520,1495,1369,1265,1234,1095 \mathrm{~cm}^{-1}$; HRMS (ESI+) $\mathrm{m} / \mathrm{z} 592.2176\left(\mathrm{M}+\mathrm{H}^{+}, \mathrm{C}_{32} \mathrm{H}_{34} \mathrm{NO}_{10}\right.$ requires $\mathrm{m} / \mathrm{z} 592.2183$ ).<smiles>COc1ccc(C(=O)Nc2cc3ccc(O[C@@H]4OC(C)(C)[C@@H](OC)[C@H](O)[C@H]4O)c(C)c3oc2=O)cc1-c1cccc2c1Sc1ccccc1S2</smiles>

38

$N$-(7-((2R,3R,4S,5R)-3,4-Dihydroxy-5-methoxy-6,6-dimethyltetrahydro-2H-pyran-2-yloxy)-8methyl-2-oxo-2H-chromen-3-yl)-4-methoxy-3-(thianthren-1-yl)benzamide (38). Colorless solid (51\%, 2 steps): $[\alpha]^{26}{ }_{\mathrm{D}}=-12.0^{\circ}\left(\mathrm{c}=0.82, \mathrm{CH}_{2} \mathrm{Cl}_{2}\right) ;{ }^{1} \mathrm{H}$ NMR $\left(500 \mathrm{MHz}, 20 \% \mathrm{CD}_{3} \mathrm{OD}\right.$ in $\left.\mathrm{CD}_{2} \mathrm{Cl}_{2}\right) \delta 8.81(\mathrm{~s}, 1 \mathrm{H})$, $8.73(\mathrm{~s}, 1 \mathrm{H}), 8.08(\mathrm{~d}, J=7.6 \mathrm{~Hz}, 1 \mathrm{H}), 7.80(\mathrm{~s}, 1 \mathrm{H}), 7.61(\mathrm{~d}, J=6.4 \mathrm{~Hz}, 1 \mathrm{H}), 7.56(\mathrm{~d}, J=6.4 \mathrm{~Hz}, 1 \mathrm{H}), 7.42$ - $7.16(\mathrm{~m}, 8 \mathrm{H}), 5.63(\mathrm{~s}, 1 \mathrm{H}), 4.28-4.22(\mathrm{~m}, 2 \mathrm{H}), 3.94(\mathrm{~s}, 3 \mathrm{H}), 3.61(\mathrm{~s}, 3 \mathrm{H}), 3.38(\mathrm{~d}, J=8.0 \mathrm{~Hz}, 1 \mathrm{H}), 2.95$ (s, 1H), $2.87(\mathrm{~s}, 1 \mathrm{H}), 2.30(\mathrm{~s}, 3 \mathrm{H}), 1.39(\mathrm{~s}, 3 \mathrm{H}), 1.16(\mathrm{~s}, 3 \mathrm{H}) ;{ }^{13} \mathrm{C} \mathrm{NMR}\left(125 \mathrm{MHz}, 20 \% \mathrm{CD}_{3} \mathrm{OD}\right.$ in $\left.\mathrm{CD}_{2} \mathrm{Cl}_{2}\right) \delta 165.1,160.1,159.2,155.9,153.5,149.1,138.0,136.3,136.1,135.8,135.6,129.9,129.7,129.6$, $129.2,128.8,128.5,127.8,127.6,127.4,126.0,125.7,123.9,122.0,114.1,114.0,111.1,110.7,97.8,84.2$, 78.5, 71.2, 68.6, 61.7, 55.9, 28.9, 22.3, 8.1; IR (film) $v_{\max } 3400,3055,2976,2932,2837,1709,1670,1605$, 1526, 1497, 1439, 1367, 1261, 1234, 1111, $1094 \mathrm{~cm}^{-1}$; HRMS (ESI+) $\mathrm{m} / \mathrm{z} 714.1822\left(\mathrm{M}+\mathrm{H}^{+}\right.$, $\mathrm{C}_{38} \mathrm{H}_{36} \mathrm{NO}_{9} \mathrm{~S}_{2}$ requires $\mathrm{m} / \mathrm{z} 714.1832$ ). 
General EDCI coupling procedure B: $N$-(3-Dimethylamino-propyl)- $N$ '-ethylcarbodiimide hydrochloride (2.5 eq) was added to a solution of aminocoumarin 7 (1.0 eq), carboxylic acid (2.0 eq) in $\mathrm{CH}_{2} \mathrm{Cl}_{2}$ containing $30 \%$ pyridine at $\mathrm{rt}$. The solution was stirred for $14 \mathrm{~h}$, concentrated and the residue purified via preparative TLC ( $\mathrm{SiO}_{2}, 40: 1 ; \mathrm{CH}_{2} \mathrm{Cl}_{2}$ :acetone) to afford the amide. The resulting product was dissolved in methanol containing $10 \% \mathrm{Et}_{3} \mathrm{~N}$ and stirred for $14 \mathrm{~h}$ at $\mathrm{rt}$. The mixture was concentrated and the residue was purified by preparative TLC $\left(10: 1 ; \mathrm{CH}_{2} \mathrm{Cl}_{2}\right.$ :methanol or $4: 1 ; \mathrm{CH}_{2} \mathrm{Cl}_{2}$ :actone) to afford the corresponding diol.<smiles>CO[C@H]1[C@@H](O)[C@@H](O)C(Oc2ccc3cc(NC(=O)c4ccccn4)c(=O)oc3c2C)OC1(C)C</smiles>

40

$N$-(7-((2R,3R,4S,5R)-3,4-Dihydroxy-5-methoxy-6,6-dimethyltetrahydro-2H-pyran-2-yloxy)-8methyl-2-oxo-2H-chromen-3-yl)picolinamide (40). Yellow solid (56\%, 2 steps): $\quad[\alpha]^{25}=-18.8^{\circ}(\mathrm{c}=$ 0.48, $20 \% \mathrm{MeOH}$ in $\left.\mathrm{CH}_{2} \mathrm{Cl}_{2}\right) ;{ }^{1} \mathrm{H}$ NMR (500 MHz, $20 \% \mathrm{CD}_{3} \mathrm{OD}$ in $\left.\mathrm{CD}_{2} \mathrm{Cl}_{2}\right) \delta 8.76(\mathrm{~s}, 1 \mathrm{H}), 8.66(\mathrm{~d}, J=4.5$ $\mathrm{Hz}, 1 \mathrm{H}), 8.18(\mathrm{~d}, J=7.5 \mathrm{~Hz}, 1 \mathrm{H}), 7.93(\mathrm{dt}, J=1.5,7.5 \mathrm{~Hz}, 1 \mathrm{H}), 7.54-7.49(\mathrm{~m}, 1 \mathrm{H}), 7.37(\mathrm{~d}, J=8.5 \mathrm{~Hz}$, 1H), 7.19 (d, $J=8.5 \mathrm{~Hz}, 1 \mathrm{H}), 5.53$ (d, $J=2.0 \mathrm{~Hz}, 1 \mathrm{H}), 4.14$ (dd, $J=3.5,9.5 \mathrm{~Hz}, 1 \mathrm{H}), 4.09$ (t, $J=3.5 \mathrm{~Hz}$, $1 \mathrm{H}), 3.55$ (s, 3H), $3.32(\mathrm{~d}, J=9.5 \mathrm{~Hz}, 1 \mathrm{H}), 2.27$ (s, 3H), $1.31(\mathrm{~s}, 3 \mathrm{H}), 1.08(\mathrm{~s}, 3 \mathrm{H}) ;{ }^{13} \mathrm{C} \mathrm{NMR}(125 \mathrm{MHz}$, $20 \% \mathrm{CD}_{3} \mathrm{OD}$ in $\left.\mathrm{CD}_{2} \mathrm{Cl}_{2}\right) \delta 165.0,160.8,158.1,151.1,150.6,150.3,139.5,128.7,127.4,126.4,123.8$, 123.0, 115.9, 115.5, 112.9, 100.2, 85.8, 80.2, 72.9, 70.1, 63.1, 30.2, 23.9, 9.5; IR (film) $v_{\max } 3421,3065$, 2982, 2932, 2837, 2476, 1717, 1682, 1626, 1607, 1522, 1458, 1414, 1375, 1265, 1173, 1134, 1097, 1080 $\mathrm{cm}^{-1}$; HRMS (ESI +$) \mathrm{m} / \mathrm{z} 471.1751\left(\mathrm{M}+\mathrm{H}^{+}, \mathrm{C}_{24} \mathrm{H}_{27} \mathrm{~N}_{2} \mathrm{O}_{8}\right.$ requires $\left.\mathrm{m} / \mathrm{z} 471.1767\right)$.<smiles>CO[C@H]1[C@@H](O)[C@@H](O)[C@H](Oc2ccc3cc(NC(=O)c4cccnc4)c(=O)oc3c2C)OC1(C)C</smiles>

41

$N$-(7-((2R,3R,4S,5R)-3,4-Dihydroxy-5-methoxy-6,6-dimethyltetrahydro-2H-pyran-2-yloxy)-8methyl-2-oxo-2H-chromen-3-yl)nicotinamide (41). Colorless solid (52\%, 2 steps): $[\alpha]^{25}=-16.1^{\circ}(\mathrm{c}=$ 0.31, 20\% MeOH in $\mathrm{CH}_{2} \mathrm{Cl}_{2}$ ); ${ }^{1} \mathrm{H}$ NMR (400 MHz, $\left.\mathrm{CD}_{2} \mathrm{Cl}_{2}\right) \delta 9.13(\mathrm{~s}, 1 \mathrm{H}), 8.80-8.75(\mathrm{~m}, 1 \mathrm{H}), 8.77(\mathrm{~s}$, $1 \mathrm{H}), 8.73(\mathrm{~s}, 1 \mathrm{H}), 8.20(\mathrm{dd}, J=1.6,7.2 \mathrm{~Hz}, 1 \mathrm{H}), 7.47(\mathrm{dd}, J=4.8,7.6 \mathrm{~Hz}, 1 \mathrm{H}), 7.38(\mathrm{~d}, J=8.4 \mathrm{~Hz}, 1 \mathrm{H})$, $7.21(\mathrm{~d}, J=8.4 \mathrm{~Hz}, 1 \mathrm{H}), 5.60(\mathrm{~d}, J=2.0 \mathrm{~Hz}, 1 \mathrm{H}), 4.27-4.17$ (m, 2H), 3.58 (s, 3H), $3.35(\mathrm{~d}, J=8.4 \mathrm{~Hz}$, 1H), 2.75 (s, 2H), 2.27 (s, 3H), $1.36(\mathrm{~s}, 3 \mathrm{H}), 1.12(\mathrm{~s}, 3 \mathrm{H}) ;{ }^{13} \mathrm{C}$ NMR $\left(125 \mathrm{MHz}, 20 \% \mathrm{CD}_{3} \mathrm{OD}\right.$ in $\left.\mathrm{CD}_{2} \mathrm{Cl}_{2}\right) \delta$ 166.0, 160.9, 158.3, 154.0, 151.1, 149.8, 137.2, 131.7, 127.6 (2C), 125.6, 123.0, 115.9, 115.3, 113.0, 100.1, 85.8, 80.2, 72.9, 70.1, 63.2, 30.2, 23.9, 9.5; IR (film) $v_{\max } 3394,3092,3065,2980,2928,2854,2833,1711$, 
1672, 1605, 1531, 1371, 1258, 1132, 1113, 1095, $1082 \mathrm{~cm}^{-1} ;$ HRMS $($ ESI +$) \mathrm{m} / \mathrm{z} 471.1763\left(\mathrm{M}+\mathrm{H}^{+}\right.$, $\mathrm{C}_{24} \mathrm{H}_{27} \mathrm{~N}_{2} \mathrm{O}_{8}$ requires $m / \mathrm{z}$ 471.1767).<smiles>CO[C@H]1[C@@H](O)[C@@H](O)C(Oc2ccc3cc(NC(=O)c4ccncc4)c(=O)oc3c2C)OC1(C)C</smiles>

42

$N$-(7-((2R,3R,4S,5R)-3,4-Dihydroxy-5-methoxy-6,6-dimethyltetrahydro-2H-pyran-2-yloxy)-8methyl-2-oxo-2H-chromen-3-yl)isonicotinamide (42). Yellow solid (52\%, 2 steps): $[\alpha]^{25}=-14.3^{\circ}(\mathrm{c}=$ $0.67,20 \% \mathrm{MeOH}$ in $\left.\mathrm{CH}_{2} \mathrm{Cl}_{2}\right) ;{ }^{1} \mathrm{H}$ NMR $\left(800 \mathrm{MHz}, 20 \% \mathrm{CD}_{3} \mathrm{OD}\right.$ in $\left.\mathrm{CD}_{2} \mathrm{Cl}_{2}\right) \delta 8.83-8.74(\mathrm{~m}, 3 \mathrm{H}), 7.87-$ $7.80(\mathrm{~m}, 2 \mathrm{H}), 7.42(\mathrm{~d}, J=8.0 \mathrm{~Hz}, 1 \mathrm{H}), 7.24(\mathrm{~d}, J=8.0 \mathrm{~Hz}, 1 \mathrm{H}), 5.58$ (d, $J=2.4 \mathrm{~Hz}, 1 \mathrm{H}), 4.20-4.11(\mathrm{~m}$, $2 \mathrm{H}), 3.60(\mathrm{~s}, 3 \mathrm{H}), 3.36(\mathrm{~d}, J=9.6 \mathrm{~Hz}, 1 \mathrm{H}), 2.28(\mathrm{~s}, 3 \mathrm{H}), 1.35(\mathrm{~s}, 3 \mathrm{H}), 1.12(\mathrm{~s}, 3 \mathrm{H}) ;{ }^{13} \mathrm{C} \mathrm{NMR}(200 \mathrm{MHz}$, $20 \% \mathrm{CD}_{3} \mathrm{OD}$ in $\mathrm{CD}_{2} \mathrm{Cl}_{2}$ ) $\delta 164.2,159.1,156.7,150.2$ (3C), 149.5, 141.5, 126.4, 126.0, 121.3, 121.0, 114.1, 113.4, 111.3, 98.5, 84.1, 78.6, 71.2, 68.4, 61.4, 28.5, 22.2, 7.8; IR (film) $v_{\max } 3393,3055,2982,2932$, $2835,1709,1674,1628,1607,1529,1373,1258,1134,1111,1095,1080 \mathrm{~cm}^{-1} ;$ HRMS (ESI +$) \mathrm{m} / \mathrm{z}$ $471.1748\left(\mathrm{M}+\mathrm{H}^{+}, \mathrm{C}_{24} \mathrm{H}_{27} \mathrm{~N}_{2} \mathrm{O}_{8}\right.$ requires $m / z$ 471.1767).

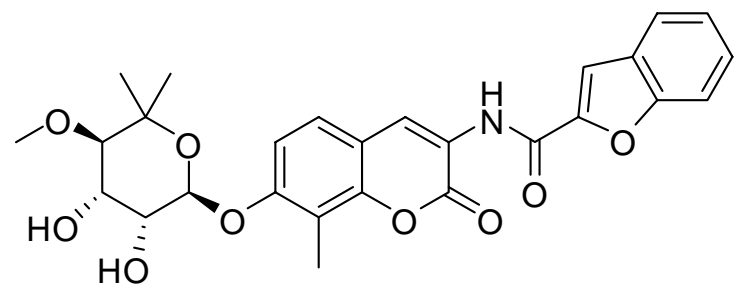

43

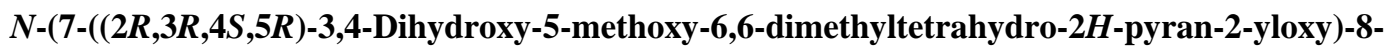
methyl-2-oxo-2H-chromen-3-yl)benzofuran-2-carboxamide (43). Colorless solid (63\%, 2 steps): $[\alpha]^{24}$ $=-16.8^{\circ}\left(\mathrm{c}=0.19,20 \% \mathrm{MeOH}\right.$ in $\left.\mathrm{CH}_{2} \mathrm{Cl}_{2}\right) ;{ }^{1} \mathrm{H} \mathrm{NMR}\left(400 \mathrm{MHz}, 20 \% \mathrm{CD}_{3} \mathrm{OD}\right.$ in $\left.\mathrm{CD}_{2} \mathrm{Cl}_{2}\right) \delta 8.70(\mathrm{~s}, 1 \mathrm{H})$, $7.68(\mathrm{dd}, J=0.8,8.8 \mathrm{~Hz}, 1 \mathrm{H}), 7.59(\mathrm{dd}, J=0.8,8.8 \mathrm{~Hz}, 1 \mathrm{H}), 7.58(\mathrm{~s}, 1 \mathrm{H}), 7.45(\mathrm{dt}, J=0.8,7.6 \mathrm{~Hz}, 1 \mathrm{H})$, $7.37(\mathrm{~d}, J=8.8 \mathrm{~Hz}, 1 \mathrm{H}), 7.29(\mathrm{dt}, J=0.8,7.6 \mathrm{~Hz}, 1 \mathrm{H}), 7.19(\mathrm{~d}, J=8.8 \mathrm{~Hz}, 1 \mathrm{H}), 5.52(\mathrm{~d}, J=2.4 \mathrm{~Hz}, 1 \mathrm{H})$, $4.12(\mathrm{dd}, J=3.4,9.2 \mathrm{~Hz}, 1 \mathrm{H}), 4.07(\mathrm{dd}, J=3.4,9.2 \mathrm{~Hz}, 1 \mathrm{H}), 3.54(\mathrm{~s}, 3 \mathrm{H}), 3.30$ (d, J=9.2 Hz, $1 \mathrm{H}), 2.25$ (s, $3 \mathrm{H}), 1.29(\mathrm{~s}, 3 \mathrm{H}), 1.06(\mathrm{~s}, 3 \mathrm{H}) ;{ }^{13} \mathrm{C}$ NMR (125 MHz, 20\% $\mathrm{CD}_{3} \mathrm{OD}$ in $\left.\mathrm{CD}_{2} \mathrm{Cl}_{2}\right) \delta 161.5,159.8,159.0,157.5$, $151.8,150.0,130.1,129.9,128.3,127.8,126.4,125.2,123.3,116.6,116.0,114.4,114.2,113.7,100.9$, 86.5, 81.0, 73.6, 70.8, 63.8, 30.8, 24.6, 10.2; IR (film) $v_{\max } 3404,3385,2986,2935,2511,1717,1670$, 1626, 1607, 1576, 1548, 1418, 1377, 1265, 1115, $1092 \mathrm{~cm}^{-1}$; HRMS (ESI+) m/z 532.1566 $\left(\mathrm{M}^{+} \mathrm{Na}^{+}\right.$, $\mathrm{C}_{27} \mathrm{H}_{27} \mathrm{NO}_{9} \mathrm{Na}$ requires $\mathrm{m} / \mathrm{z} 532.1583$ ). 


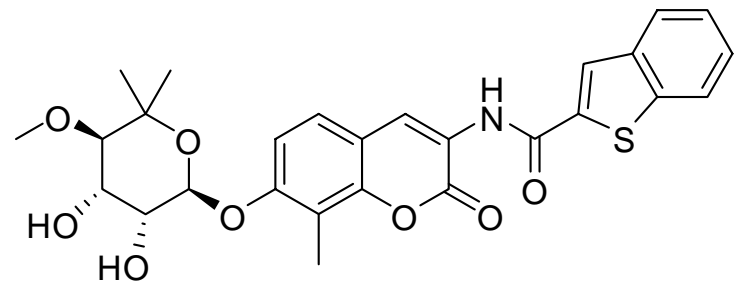

44

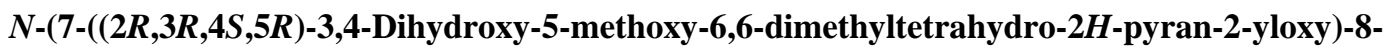
methyl-2-oxo-2H-chromen-3-yl)benzo[b]thiophene-2-carboxamide (44). Colorless solid (42\%, 2 steps): $[\alpha]_{\mathrm{D}}^{25}=-24.3^{\circ}\left(\mathrm{c}=0.23,20 \% \mathrm{MeOH}\right.$ in $\left.\mathrm{CH}_{2} \mathrm{Cl}_{2}\right) ;{ }^{1} \mathrm{H}$ NMR $\left(400 \mathrm{MHz}, 20 \% \mathrm{CD}_{3} \mathrm{OD}\right.$ in $\left.\mathrm{CD}_{2} \mathrm{Cl}_{2}\right) \delta 8.66(\mathrm{~s}$, 1H), $8.00(\mathrm{~s}, 1 \mathrm{H}), 7.88$ (t, $J=7.4 \mathrm{~Hz}, 2 \mathrm{H}), 7.45-7.33(\mathrm{~m}, 3 \mathrm{H}), 7.19$ (d, $J=8.8 \mathrm{~Hz}, 1 \mathrm{H}), 5.53(\mathrm{~d}, J=2.0$ Hz, 1H), 4.12 (d, J = 9.2 Hz, 1H), $4.09-4.05$ (m, 1H), 3.54 (s, 3H), 3.31 (d, J = 9.2 Hz, 1H), 2.25 (s, 3H), $1.30(\mathrm{~s}, 3 \mathrm{H}), 1.07(\mathrm{~s}, 3 \mathrm{H}) ;{ }^{13} \mathrm{C} \mathrm{NMR}\left(125 \mathrm{MHz}, 20 \% \mathrm{CD}_{3} \mathrm{OD}\right.$ in $\left.\mathrm{CD}_{2} \mathrm{Cl}_{2}\right) \delta 161.6,156.9,149.0,145.3$, $141.8,139.5$, 138.0, 127.2, 126.6, 126.2, 126.0, 125.8, 125.5, 123.0, 121.3, 114.7, 114.2, 111.7, 98.9, 84.5, 78.9, 71.6, 68.8, 61.7, 28.8, 22.6, 8.1; IR (film) $v_{\max } 3414,3381,2986,2932,2526,1690,1649,1632$, 1601, 1529, 1375, 1254, 1240, 1194, $1084 \mathrm{~cm}^{-1}$; HRMS (ESI+) m/z $526.1532\left(\mathrm{M}+\mathrm{H}^{+}, \mathrm{C}_{27} \mathrm{H}_{28} \mathrm{NO}_{8} \mathrm{~S}\right.$ requires $m / z$ 526.1536).<smiles>CO[C@H]1[C@@H](O)[C@@H](O)[C@H](Oc2ccc3cc(NC(=O)c4ccc5c(c4)OCO5)c(=O)oc3c2C)OC1(C)C</smiles>

45

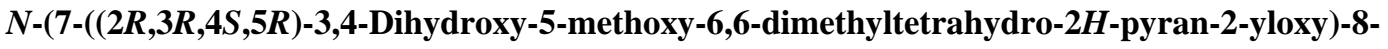
methyl-2-oxo-2H-chromen-3-yl)benzo[d][1,3]dioxole-5-carboxamide (45). Colorless solid (39\%, 2 steps): $[\alpha]^{26}=-15.9^{\circ}\left(\mathrm{c}=0.27,20 \% \mathrm{MeOH}\right.$ in $\left.\mathrm{CH}_{2} \mathrm{Cl}_{2}\right) ;{ }^{1} \mathrm{H} \mathrm{NMR}\left(500 \mathrm{MHz}, 20 \% \mathrm{CD}_{3} \mathrm{OD}\right.$ in $\left.\mathrm{CD}_{2} \mathrm{Cl}_{2}\right) \delta$ $8.64(\mathrm{~s}, 1 \mathrm{H}), 7.39$ (d, $J=8.0 \mathrm{~Hz}, 1 \mathrm{H}), 7.31(\mathrm{~s}, 1 \mathrm{H}), 7.30(\mathrm{~d}, J=8.0 \mathrm{~Hz}, 1 \mathrm{H}), 7.14(\mathrm{~d}, J=8.5 \mathrm{~Hz}, 1 \mathrm{H}), 6.84$ $(\mathrm{d}, J=8.5 \mathrm{~Hz}, 1 \mathrm{H}), 5.99(\mathrm{~s}, 2 \mathrm{H}), 5.48(\mathrm{~s}, 1 \mathrm{H}), 4.08(\mathrm{~d}, J=9.5 \mathrm{~Hz}, 1 \mathrm{H}), 4.04(\mathrm{~d}, J=2.0 \mathrm{~Hz}, 1 \mathrm{H}), 3.48$ (s, $3 \mathrm{H}), 3.25(\mathrm{~d}, J=9.5 \mathrm{~Hz}, 1 \mathrm{H}), 2.20(\mathrm{~s}, 3 \mathrm{H}), 1.22(\mathrm{~s}, 3 \mathrm{H}), 1.12(\mathrm{~s}, 3 \mathrm{H}) ;{ }^{13} \mathrm{C} \mathrm{NMR}\left(125 \mathrm{MHz}, 20 \% \mathrm{CD}_{3} \mathrm{OD}\right.$ in $\left.\mathrm{CD}_{2} \mathrm{Cl}_{2}\right) \delta 164.7,158.7,155.6,150.6,148.4,147.7,127.2,125.1,123.9,121.5,121.0,113.5,113.2,110.6$, 107.5, 106.8, 101.5, 97.7, 83.5, 77.9, 70.5, 67.8, 60.8, 27.9, 21.6, 7.2; IR (film) $v_{\max } 3404,3111,3035$, 2980, 2926, 2853, 1701, 1607, 1528, 1485, 1444, 1406, 1369, 1254, 1132, 1113, 1086, $1038 \mathrm{~cm}^{-1}$; HRMS $(\mathrm{ESI}+) \mathrm{m} / \mathrm{z} 514.1708\left(\mathrm{M}+\mathrm{H}^{+}, \mathrm{C}_{26} \mathrm{H}_{28} \mathrm{~N}_{2} \mathrm{O}_{10}\right.$ requires $\mathrm{m} / \mathrm{z}$ 514.1713). 
<smiles>CO[C@H]1[C@@H](O)[C@@H](O)[C@H](Oc2ccc3cc(NC(=O)c4cc5ccccc5[nH]4)c(=O)oc3c2C)OC1(C)C</smiles>

46

$N$-(7-((2R,3R,4S,5R)-3,4-Dihydroxy-5-methoxy-6,6-dimethyltetrahydro-2H-pyran-2-yloxy)-8methyl-2-oxo- $2 H$-chromen-3-yl)-1H-indole-2-carboxamide (46). Yellow solid (31\%, 2 steps): $[\alpha]_{D}^{25}=$ $-18.2^{\circ}\left(\mathrm{c}=0.22,20 \% \mathrm{MeOH}\right.$ in $\left.\mathrm{CH}_{2} \mathrm{Cl}_{2}\right) ;{ }^{1} \mathrm{H}$ NMR $\left(800 \mathrm{MHz}, 20 \% \mathrm{CD}_{3} \mathrm{OD}\right.$ in $\left.\mathrm{CD}_{2} \mathrm{Cl}_{2}\right) \delta 8.69(\mathrm{~s}, 1 \mathrm{H}), 7.66$ (d, $J=8.0 \mathrm{~Hz}, 1 \mathrm{H}), 7.46$ (d, $J=8.0 \mathrm{~Hz}, 1 \mathrm{H}), 7.36$ (d, $J=9.0 \mathrm{~Hz}, 1 \mathrm{H}), 7.27$ (t, $J=7.5 \mathrm{~Hz}, 1 \mathrm{H}), 7.20$ (d, $J=$ $9.0 \mathrm{~Hz}, 1 \mathrm{H}), 7.17$ (s, 1H), 7.10 (t, J=7.5 Hz, 1H), 5.53 (d, J = 2.0 Hz, 1H), $4.17-4.08$ (m, 2H), 3.55 (s, $3 \mathrm{H}), 3.31(\mathrm{~d}, J=9.5 \mathrm{~Hz}, 1 \mathrm{H}), 2.26(\mathrm{~s}, 3 \mathrm{H}), 1.30(\mathrm{~s}, 3 \mathrm{H}), 1.08(\mathrm{~s}, 3 \mathrm{H}) ;{ }^{13} \mathrm{C}$ NMR $\left(200 \mathrm{MHz}, 20 \% \mathrm{CD}_{3} \mathrm{OD}\right.$ in $\left.\mathrm{CD}_{2} \mathrm{Cl}_{2}\right) \delta 162.1,161.0,158.0,150.9,139.0,131.8,129.1,127.3,126.6(2 \mathrm{C}), 123.7,123.1,122.3,115.8$, 115.5, 113.7, 112.9, 106.9, 100.2, 85.8, 80.2, 72.9, 70.1, 63.1, 30.1, 23.9, 9.4; IR (film) $v_{\max } 3443,3421$, 3404, 3003, 2986, 2935, 1609, 1541, 1364, 1263, 1105, $1082 \mathrm{~cm}^{-1}$; HRMS (ESI+) $\mathrm{m} / \mathrm{z} 509.1916\left(\mathrm{M}+\mathrm{H}^{+}\right.$, $\mathrm{C}_{27} \mathrm{H}_{29} \mathrm{~N}_{2} \mathrm{O}_{8}$ requires $m / z$ 509.1924).<smiles>CO[C@H]1[C@@H](O)[C@@H](O)C(Oc2ccc3cc(NC(=O)c4c[nH]c5ccccc45)c(=O)oc3c2C)OC1(C)C</smiles>

47

$N$-(7-((2R,3R,4S,5R)-3,4-Dihydroxy-5-methoxy-6,6-dimethyltetrahydro-2H-pyran-2-yloxy)-8methyl-2-oxo-2H-chromen-3-yl)-1H-indole-3-carboxamide (47). Colorless solid (19\%, 2 steps): $[\alpha]^{26}$ $=-11.4^{\circ}\left(\mathrm{c}=0.18,20 \% \mathrm{MeOH}\right.$ in $\left.\mathrm{CH}_{2} \mathrm{Cl}_{2}\right) ;{ }^{1} \mathrm{H}$ NMR $\left(500 \mathrm{MHz}, 20 \% \mathrm{CD}_{3} \mathrm{OD}\right.$ in $\left.\mathrm{CD}_{2} \mathrm{Cl}_{2}\right) \delta 8.75(\mathrm{~s}, 1 \mathrm{H})$, 8.12 (dd, $J=2.0,6.5 \mathrm{~Hz}, 1 \mathrm{H}), 7.97$ (s, 1H), 7.49 (dd, $J=2.0,6.5 \mathrm{~Hz}, 1 \mathrm{H}), 7.38$ (d, $J=8.5 \mathrm{~Hz}, 1 \mathrm{H}), 7.31$ $7.24(\mathrm{~m}, 2 \mathrm{H}), 7.20$ (d, $J=8.5 \mathrm{~Hz}, 1 \mathrm{H}), 5.55(\mathrm{~d}, J=2.5 \mathrm{~Hz}, 1 \mathrm{H}), 4.16$ (dd, $J=4.5,9.5 \mathrm{~Hz}, 1 \mathrm{H}), 4.12$ (t, $J=$ $4.5 \mathrm{~Hz}, 1 \mathrm{H}), 3.57(\mathrm{~s}, 3 \mathrm{H}), 3.33-3.31(\mathrm{~m}, 1 \mathrm{H}), 2.28(\mathrm{~s}, 3 \mathrm{H}), 1.33(\mathrm{~s}, 3 \mathrm{H}), 1.10(\mathrm{~s}, 3 \mathrm{H}) ;{ }^{13} \mathrm{C} \mathrm{NMR}(125$ $\mathrm{MHz}, 20 \% \mathrm{CD}_{3} \mathrm{OD}$ in $\left.\mathrm{CD}_{2} \mathrm{Cl}_{2}\right) \delta 165.7,161.3,157.6,150.6,138.4,131.0,127.1,126.4,125.1,124.5$, 123.8, 123.5, 121.4, 115.8 (2C), 113.9, 112.8, 112.7, 100.0, 85.9, 80.1, 72.9, 70.1, 63.2, 30.2, 23.9, 9.6; IR (film) $v_{\max } 3439,3418,3394,2957,2924,2853,1636,1529,1437,1379,1261,1180,1128,1082,1020 \mathrm{~cm}^{-}$ 1; HRMS (ESI+) m/z $509.1924\left(\mathrm{M}+\mathrm{H}^{+}, \mathrm{C}_{27} \mathrm{H}_{29} \mathrm{~N}_{2} \mathrm{O}_{8}\right.$ requires $\mathrm{m} / \mathrm{z}$ 509.1924). 